\title{
ON THE LATTICE BOLTZMANN DEVIATORIC STRESS: ANALYSIS, BOUNDARY CONDITIONS, AND OPTIMAL RELAXATION TIMES
}

\author{
T. REIS*
}

\begin{abstract}
.
We analytically solve the two dimensional, nine-velocity, lattice Boltzmann model in planar channel flow and determine its deviatoric stress tensor. The shear component of its stress takes the expected Navier-Stokes form but the tangential component contains second order in Knudsen number contributions that one finds in solutions to the Burnett equations. Boundary conditions that neglect this Burnett contribution cause spurious grid-scale oscillations in the computed stress field within the computational domain. A moment-based boundary condition which considers the non-zero deviatoric stress is analysed and shown to completely eliminate the spurious oscillations seen in solutions using other boundary conditions. The analysis offers an explanation of previously reported optimal relaxation times in terms of the recurrence relation for the tangential stress and gives them an interpretation in terms of compact finite difference schemes.
\end{abstract}

Key words. Lattice Boltzmann equation, Burnett stress, compact finite difference schemes, boundary conditions, two relaxation time models

The lattice Boltzmann equation (LBE) is a numerical algorithm derived from a velocityspace truncation of Boltzmann's equation for monatomic gases [23]. Despite being an algorithm that computes at each discrete point in space and time a discrete velocity distribution function it is primarily used to numerically solve the Navier-Stokes equations, the variables of which are obtained from moments of the distribution functions. The kinetic heritage of the LBE has encouraged boundary conditions for the algorithm to be formulated in terms of this particle basis, where the unknown distribution functions are usually found by "bounce-back" $[11,25]$ - a reversal in the velocity distribution functions that hit a boundary - or an adaptation of Maxwell's combination of diffuse and specular reflection [32] to a discrete velocity space. Bounce-back methods have been successful extended to complex geometries of engineering importance where physical boundaries are not necessarily aligned with lattice gridpoints, usually by combining them with spatial interpolations to ensure macroscopic conditions on the velocity are satisfied [5, 13, 48, 52]. Diffuse boundary conditions were first applied to discrete velocity Boltzmann models by Broadwell [6] and studied in detail by Gatignol [15] before being discussed in terms of the lattice Boltzmann method [2]. Another popular method is "non-equilibrium bounce-back" [53] which, unlike bounce-back and Maxwell-Broadwell conditions, explicitly imposes a macroscopic wall velocity condition.

The supposed simplicity of "particle-based" boundary conditions is often regarded as one of the LBE's major advantages over traditional (macroscopic) numerical methods, yet it has been a source of much debate. This is due, in part at least, to the indirect approach of using a discrete kinetic-based model to solve the hydrodynamic flow equations: on the one hand, boundary conditions are needed for the particle velocity distribution functions; while on the other, accurate solutions of the macroscopic flow variables are usually sought. Moreover, the flow behaviour in the vicinity of solid boundaries is considerably different in kinetic flows compared with hydrodynamic flows when the Knudsen number $(K n)$ is appreciable. For example, kinetic theory can accurately predict the Knudsen layer in the slip-flow regime an $\mathcal{O}(K n)$-wide boundary layer in the vicinity of the wall [7, 41] - whereas Navier-Stokes theory cannot [21]. The lattice Boltzmann equation with either bounce-back or MaxwellBroadwell conditions usually predicts a non-vanishing fluid velocity at wall boundaries. This apparent slip - sometimes presumed to be a kinetic effect - has stimulating much interest in the applicability of the LBE - primarily a hydrodynamic flow solver - to the rarefied flow

${ }^{*}$ School of Computing and Mathematical Sciences, University of Greenwich SE10 9LS(T.Reisegreenwich.ac.uk). 
regime, but equally as much controversy $[2,50,29,44,43,38,37,25,46]$.

The numerical and physical nature of the lattice Boltzmann equation can be elucidated with simple, analytically tractable, flows from which we can obtain exact solutions. He et al. [25] analytically solved the D2Q9 BGK lattice Boltzmann equation for the velocity field in planar channel flow. They showed that the LBE reduces in this flow to a three-term recurrence relation for the flow velocity, the solution of which is a perfect parabolic profile, as in Poiseuille flow, with a constant numerical (artificial) slip velocity, $U_{s}$, that is determined by the boundary conditions. More specifically, the position where the tangential velocity vanishes only asymptotically coincides with the point halfway between grid points; its precise location depending on the kinematic viscosity. The general form of the solution is valid for all boundary conditions and all Knudsen numbers, and hence one concludes that the D2Q9 lattice Boltzmann equation does not predict kinetic effects in the velocity field. We remark briefly that the numerical slip velocity stems from the particles moving tangentially along the wall and will be present in any boundary condition implementation that does not fully account for them. Complications from so-called 'grazing' molecules have been known to exist in discrete kinetic theory for some time [15],

The D2Q9 numerical slip may be eliminated with two relaxation time (TRT) models provided the so-called "magic relation" between the relaxation times for the odd and even moments is satisfied, $\Lambda=3 / 16[16,18,10,34,46]$. d'Humieres and Ginzburg [10] analysed the lattice Boltzmann recurrence equation with two relaxation times in steady flows and showed also that the numerical errors and stability of the algorithm are controlled by the product of odd and even relaxation times, rather than on each individually. Thus the "magic parameter" $\Lambda$ is more than just a fix for the slip artefact in a simple flow. Unfortunately, the choice of $\Lambda$ which eliminates the numerical-slip does not coincide with the most stable or apparently optimal set of relaxation times [17, 10, 26, 12].

The inability of the D2Q9 LBE to predict Knudsen boundary layers in the velocity field does not preclude more subtle kinetic effects. In classical kinetic theory one can obtain the Burnett equations from the Chapman-Enskog expansion at $\mathcal{O}\left(K n^{2}\right)$ with a fixed Mach $(M a)$ number $[8,14]$ and equations of Grad-type are obtained from a Hermite polynomial closure [20]. Neither the Burnett nor Grad 13 equations (which are both formally of second order in Knudsen number) can capture kinetic boundary layers in the velocity field, but rarefaction effects manifest themselves in these models as an $\mathcal{O}\left(K n^{2}\right)\left(\right.$ or $\left.\mathcal{O}\left(\tau^{2}\right)\right)$ contribution to higher moments [42]. Interestingly, Yudisiawan [49] observed some kinetic effects in the stress field of the D2Q9 discrete Boltzmann model: solutions of the truncated PDE moment system suggested a non zero tangential stress in planar (force-driven) Poiseuille flow; something which is characteristic of Burnett and non-Newtonian behaviour. More recently, Dellar [9] took inspiration from [27] and [45] and showed the constitutive equation for stress embedded within the moments of the D2Q9 discrete Boltzmann equations is not that of the Navier-Stokes equations and resembles the upper convected Maxwell model for viscoelasticity. Reis [36] also noticed that that the LBE predicted non-Newtonian behaviour in the stress field and used the work of Dellar [9] to develo suitable boundary conditions for the tangential component of stress. Although Yong and Luo [47] showed that the D2Q9 lattice Boltzmann equation predicts the Newtonian viscous stress tensor with second order accuracy, their calculations assume a diffusive scaling where the timestep is proportional to the square of the grid spacing, $\Delta t \propto \Delta x^{2}$. This suppresses acoustic behaviour and kinetic effects at $\mathcal{O}\left(\tau^{2}\right)$.

The kinetic or non-Newtonian effects that appear to be embedded in the lattice Boltzmann equation can manifest near the boundaries, as well as in the flow, implying that boundary conditions for the LBE may need to be informed of the $\mathcal{O}\left(\tau^{2}\right)$ terms in the stress. Most existing lattice Boltzmann implementations of boundary conditions do not have the freedom to do this, as will be discussed in Section 2. An exception is the moment-based method of 
Bennett [3]. Most existing work using this approach assumes the stress is of Navier-Stokes form $[36,37,1,22,33]$, which may be inconsistent with the underlying PDE moment system, but this can be adjusted. Reis [36] showed numerically that the Navier-Stokes assumption in the boundary conditions causes spurious oscillations in the tangential component of the deviatoric stress and proposed a consistent constraint, but no further analysis of the algorithm was provided.

In this article we take the view that the lattice Boltzmann equation is a direct space-time discretisation of the discrete velocity Boltzmann equation with a truncated (closed) moment PDE system, and is thus computing solutions to this moment system. The moment system has embedded within it a non-Newtonian constitutive equation for the deviatoric stress. We hypothesise that standard local lattice Boltzmann boundary conditions that prescribe hydrodynamic conditions but do not fully account for the stress embedded within the moments are inconsistent with the underlying PDE and produce spurious oscillations in the numerical solutions to simple flows. We build on the work of He et al. [25],D'Humieres and Ginzburg [10], Dellar [9], and Reis [36], and solve the BGK and TRT lattice Boltzmann equation analytically for the three components of the stress tensor in force driven Poiseuille flow in an infinitely long channel (no inflow/outflow conditions). This allows us to see precisely how the LBE is behaving and illuminates some physical and numerical aspects of the algorithm. That is, we determine what stress the LBE is computing, the stencil it uses to compute it, the role of the relaxation times on the numerics, and the consistency of boundary conditions. The remainder of this article is organised as follows. Section 1 discusses the discrete Boltzmann PDE and the lattice Boltzmann implementation. Section 2 reviews commonly used boundary conditions and reveals their inconsistencies with the Boltzmann stress. In Section 3 we obtain the analytical solution of the lattice Boltzmann stress field in planar channel flow and discuss the Burnett boundary condition in Section 4. The significance of two-relaxation-time models is addressed in Section 5 and concluding remarks are made in Section 6.

1. The discrete Boltzmann equation and its moment PDE system. The discrete Boltzmann equation

$$
\frac{\partial f_{i}}{\partial t}+\boldsymbol{\xi}_{i} \cdot \nabla f_{i}=-\frac{1}{\tau}\left(f_{i}-f_{i}^{(0)}\right)+S_{i},
$$

describes the spatial and temporal evolution of the distribution of particles in a monatomic gas with velocity restricted to a discrete, finite, set. For the remainder of this article we focus our attention on the D2Q9 lattice [35] shown in Figure 1. The left hand side of equation (1.1) models the advection of $f_{i}$ with discrete velocity $\boldsymbol{\xi}_{i}$ and defines a linear, constant coefficient, hyperbolic, system of equations with characteristic velocities equal to $\boldsymbol{\xi}_{i}$. Boundary conditions should supply values of $f_{i}$ along these characteristics and into the domain. The first term on the right hand side is algebraic and approximates the repeated action of particle collisions, which is an assumed relaxation to the local equilibria $f_{i}^{(0)}$ with a single relaxation time $\tau$ (BGK operator). The source term $S_{i}$ can account for an additional body force. Macroscopic quantities are defined through the discrete moments of $f_{i}$. The first six of these correspond to the hydrodynamic quantities of density (a scalar), momentum (a vector), and the symmetric moment flux tensor,

$$
\rho=\sum_{i} f_{i}, \quad \rho u_{\alpha}=\sum_{i} f_{i} \xi_{\alpha}, \quad \Pi_{\alpha \beta}=\sum_{i} f_{i} \xi_{\alpha} \xi_{\beta},
$$

where the Greek subscripts refer to the Cartesian coordinates of space. The 9 dimensional particle velocity basis permits 9 independent moments. The remaining three are often called kinetic moments, but for the D2Q9 model they may also be dubbed "ghost" moments since 
they do not have a direct physical interpretation:

$$
Q_{x x y}=\sum_{i} f_{i} \xi_{i x}^{2} \xi_{i y}, \quad Q_{x y y}=\sum_{i} f_{i} \xi_{i x} \xi_{i y}^{2}, \quad R_{x x y y}=\sum_{i} f_{i} \xi_{i x}^{2} \xi_{i y}^{2} .
$$

The most commonly used equilibria $f_{i}^{(0)}$ are given by [35, 23]

$$
f_{i}^{(0)}=w_{i} \rho\left(1+\frac{\boldsymbol{\xi}_{i} \cdot \mathbf{u}}{c_{s}^{2}}+\frac{\left(\boldsymbol{\xi}_{i} \cdot \mathbf{u}\right)^{2}}{2 c_{s}^{4}}-\frac{|\mathbf{u}|^{2}}{2 c_{s}^{2}}\right),
$$

where the sound speed $c_{s}$ and weights $w_{i}$ are constants. The D2Q9 discrete velocity set is defined by

$$
\boldsymbol{\xi}_{i}=\left\{\begin{aligned}
(0,0), & i & =0, \\
\left(\cos \alpha_{i}, \sin \alpha_{i}\right) c, & i & =1,2,3,4, \\
\sqrt{2}\left(\cos \alpha_{i}, \sin \alpha_{i}\right) c, & i & =5,6,7,8,
\end{aligned}\right.
$$

where $\alpha_{i}=(i-1) \pi / 2$ for $i=1, \ldots, 4$ and $\alpha_{i}=(i-5) \pi / 2+\pi / 4$ for $i=4, \ldots, 8$ and the weights are

$$
w_{i}=\left\{\begin{array}{rlrl}
4 / 9, & & i=0, \\
1 / 9, & i & =1,2,3,4, \\
1 / 36, & i=5,6,7,8 .
\end{array}\right.
$$

These $w_{i}$ and $\boldsymbol{\xi}_{i}$ correspond to a 5th-order Gauss-Hermite quadrature [23, 39]. In these units, $c_{s}=1 / \sqrt{3}$. The first three moments of $f_{i}^{(0)}$ give the mass and momentum, i.e. they are conserved under collisions:

$$
\sum_{i} f_{i}^{(0)}=\sum_{i} f_{i}=\rho, \quad \sum_{i} f_{i}^{(0)} \xi_{\alpha}=\sum_{i} f_{i} \xi_{\alpha}=\rho u_{\alpha},
$$

and the equilibrium momentum flux tensor is

$$
\Pi_{\alpha \beta}^{(0)}=\sum_{i} f_{i}^{(0)} \xi_{\alpha} \xi_{\beta}=c_{s}^{2} \rho \delta_{\alpha \beta}+\rho u_{\alpha} u_{\beta},
$$

where the first term on the right-hand side is an ideal equation of state for the (thermodynamic) pressure. The three remaining equilibrium moments are

$$
Q_{x x y}^{(0)}=c_{s}^{2} \rho u_{y}, \quad Q_{x y y}^{(0)}=c_{s}^{2} \rho u_{x}, \quad R_{x x y y}^{(0)}=c_{s}^{4} \rho+c_{s}^{2} \rho\left(u_{x}^{2}+u_{y}^{2}\right) .
$$

1.1. Constitutive equation from the discrete Boltzmann equation. Taking successive moments of the discrete Boltzmann equation (1.1) leads to a truncated system of partial differential equations. If we ignore the force term $S_{i}$ for the time being (this will be addressed in Section 1.2), the zeroth, first and second order moment equations correspond to the mathematical statements of mass and momentum conservation, and the evolution of the momentum flux, respectively:

$$
\begin{aligned}
& \frac{\partial \rho}{\partial t}+\nabla \cdot \rho \mathbf{u}=0, \\
& \frac{\partial \rho \mathbf{u}}{\partial t}+\nabla \cdot \boldsymbol{\Pi}=0, \\
& \frac{\partial \boldsymbol{\Pi}}{\partial t}+\nabla \cdot \mathbf{Q}=-\frac{1}{\tau}\left(\boldsymbol{\Pi}-\boldsymbol{\Pi}^{(0)}\right) .
\end{aligned}
$$




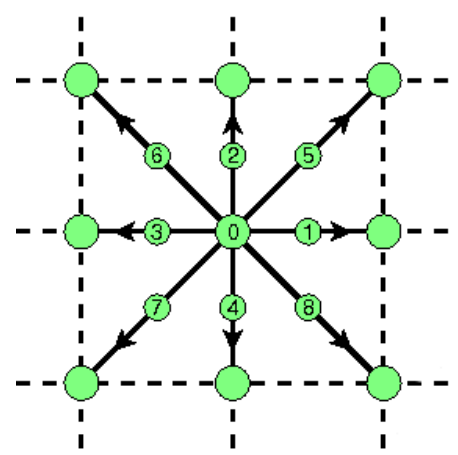

FIG. 1. (colour online) The nine particle propagation velocities $\boldsymbol{\xi}_{0}, \ldots, \boldsymbol{\xi}_{8}$ in the D2Q9 integer lattice.

The macroscopic equations of motion are most commonly obtained from the Boltzmann equation using the Chapman-Enskog expansion [8], which seeks solutions which vary slowly over timescales much longer than the collision time $\tau$. Alternatively, one may obtain an equation for the stress deviator $\mathbf{T}=\Pi^{(0)}-\Pi$ from Maxwell's equations of transfer by taking moments with respect to the peculiar velocity $\mathbf{c}_{i}=\boldsymbol{\xi}_{i}-\mathbf{u}$ [27]. The left hand side of equation (1.12) becomes

$$
\begin{aligned}
\partial_{t} \Pi_{\alpha \beta}+\partial_{\gamma} Q_{\alpha \beta \gamma} & =\partial_{t}\left(\Pi_{\alpha \beta}^{(0)}-T_{\alpha \beta}\right) \\
& +\partial_{\gamma}\left[\mathcal{Q}_{\alpha \beta \gamma}+u_{\alpha}\left(P \delta_{\beta \gamma}-T_{\beta \gamma}\right)+u_{\beta}\left(P \delta_{\gamma \alpha}-T_{\gamma \alpha}\right)\right. \\
& \left.+u_{\gamma}\left(P \delta_{\alpha \beta}-T_{\alpha \beta}\right)+\rho u_{\alpha} u_{\beta} u_{\gamma}\right]
\end{aligned}
$$

where $\mathcal{Q}_{\alpha \beta \gamma}=\sum_{i} f_{i} c_{i \alpha} c_{i \beta} c_{i \gamma}$ and $P=\rho / 3$ is the pressure. We use the conservation equations for mass (1.10) and momentum (1.11) to evaluate the temporal derivative,

$$
\partial_{t}\left(\rho u_{\alpha} u_{\beta}\right)=-u_{\alpha} \partial_{\gamma}\left(\Pi_{\beta \gamma}^{(0)}-T_{\beta \gamma}\right)-u_{\beta} \partial_{\gamma}\left(\Pi_{\alpha \gamma}^{(0)}-T_{\alpha \gamma}\right)+u_{\alpha} u_{\beta} \partial_{\gamma}\left(\rho u_{\gamma}\right) .
$$

The equilibrium part of the third order moment with respect to the peculiar velocity is

$$
\mathcal{Q}_{\alpha \beta \gamma}^{(0)} \propto \mathcal{O}\left(M a^{3}\right) .
$$

If we assume $\mathcal{Q}_{\alpha \beta \gamma} \approx \mathcal{Q}_{\alpha \beta \gamma}^{(0)}$, which is justifiable with a suitable collision operator that has a short relaxation time for $\mathbf{Q}$ then we find the evolution equation of the deviatoric stress $T_{\alpha \beta}$ from equation (1.12):

$$
T_{\alpha \beta}+\tau\left[\partial_{t} T_{\alpha \beta}+u_{\gamma} \partial_{\gamma} T_{\alpha \beta}+T_{\alpha \gamma} \frac{\partial u_{\beta}}{\partial \gamma}+T_{\beta \gamma} \frac{\partial u_{\alpha}}{\partial \gamma}\right]=\tau \theta \rho\left(\frac{\partial u_{\alpha}}{\partial \beta}+\frac{\partial u_{\beta}}{\partial \alpha}\right),
$$

where we have neglected terms of order $\mathcal{O}\left(M a^{2}\right)$ (and the Mach number $M a=|\mathbf{u}| / c_{s} \ll 1$ ). For the case of steady unidirectional channel flow, which is the primary focus of the remainder of this article, the three components of equation (1.15) simplify to

$$
T_{x x}=-2 \mu \tau\left(u_{x}^{\prime}\right)^{2}, \quad T_{x y}=\mu u_{x}^{\prime}, \quad T_{y y}=0,
$$

where $\mu=\rho \nu=\rho \tau c_{s}^{2}$ is the dynamic viscosity and primes denote differentiation with respect to $y$. 
Equation (1.16) highlights the behaviour of the deviatoric stress found from the D2Q9 discrete Boltzmann equation. At first order in Knudsen number (or $\tau$ ), $T_{x x}=T_{x x}^{(0)}=0$, giving the isothermal Navier-Stokes equations, but at the next order (the Burnett level) the tangential component of $\boldsymbol{T}$ is proportional to the square of the shear rate.

1.2. Lattice Boltzmann implementation. Equation (1.1) may be fully discretised by integrating along a characteristic for time $\Delta t$ :

$$
f_{i}\left(\mathbf{x}+\boldsymbol{\xi}_{i} \Delta t, t+\Delta t\right)-f_{i}(\mathbf{x}, t)=\int_{0}^{\Delta t} \mathcal{C}_{i}\left(x+\xi_{i} s, t+s\right) d s,
$$

where $\mathcal{C}_{i}$ represents the collision operator and body force on the right-hand side of (1.1). Approximating the right hand side of (1.17) using the trapezoidal rule gives

$$
f_{i}\left(\mathbf{x}+\boldsymbol{\xi}_{i} \Delta t, t+\Delta t\right)-f_{i}(\mathbf{x}, t)=\frac{\Delta t}{2}\left(\mathcal{C}_{i}\left(\mathbf{x}+\boldsymbol{\xi}_{i} \Delta t, t+\Delta t\right)+\mathcal{C}_{i}(\mathbf{x}, t)\right)+\mathcal{O}\left(\Delta t^{3}\right) .
$$

Equation (1.18) is a second order accurate but implicit system of algebraic equations, since $\mathcal{C}_{i}$ depends on $f_{i}$ through $\rho$ and $\mathbf{u}$. For an explicit algorithm we follow He et al. [24] and introduce the change of variables

$$
\bar{f}_{i}(\mathbf{x}, t)=f_{i}(\mathbf{x}, t)+\frac{\Delta t}{2 \tau}\left(f_{i}(\mathbf{x}, t)-f_{i}^{(0)}(\mathbf{x}, t)\right)-\frac{\Delta t}{2} S_{i}(\mathbf{x}, t) .
$$

The lattice Boltzmann equation for $\bar{f}_{i}$ at the new timestep is (1.20)

$$
\bar{f}_{i}\left(\mathbf{x}+\boldsymbol{\xi}_{i} \Delta t, t+\Delta t\right)-\bar{f}_{i}(\mathbf{x}, t)=-\frac{\Delta t}{\tau+\Delta t / 2}\left(\bar{f}_{i}(\mathbf{x}, t)-f_{i}^{(0)}(\mathbf{x}, t)\right)+\frac{\tau \Delta t}{\tau+\Delta t / 2} S_{i} .
$$

The source term $S_{i}$ included to introduce a body force $\mathbf{F}$ to the flow is required to fulfil the following moment conditions:

$$
\sum_{i} S_{i}=0, \quad \sum_{i} S_{i} \xi_{\alpha}=F_{\alpha}, \quad \sum_{i} S_{i} \xi_{\alpha} \xi_{\alpha}=F_{\alpha} u_{\beta}+u_{\alpha} F_{\beta}
$$

The first constraint in (1.21) is a statement of mass conservation and the second accounts for an additional acceleration. The third condition ensures $\mathbf{F}$ does not appear in equation (1.15). A suitable form of $S_{i}$ based on a truncated expansion in Hermite polynomials is [31]

$$
S_{i}=w_{i}\left[\frac{\boldsymbol{\xi}_{i}-\mathbf{u}}{c_{s}^{2}}+\frac{\boldsymbol{\xi}_{i} \cdot \mathbf{u}}{c_{s}^{4}} \boldsymbol{\xi}_{i}\right] \cdot \mathbf{F} .
$$

For planar channel flow we assume a constant body force in the horizontal direction, $\mathbf{F}=$ $(\rho G, 0)$.

The density is obtained directly form the zeroth order moment of $\bar{f}_{i}$ :

$$
\rho=\sum_{i} f_{i}=\sum_{i} \bar{f}_{i} ;
$$

and the momentum from the first order moment of (1.19):

$$
\rho \overline{\mathbf{u}}=\sum_{i} \bar{f}_{i} \boldsymbol{\xi}_{i}=\rho \mathbf{u}-\frac{\Delta t}{2} \mathbf{F} .
$$




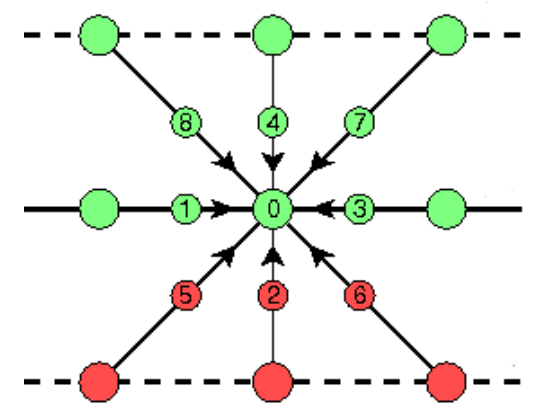

FIG. 2. (colour online) The pre-collisional states at a point on the southern boundary. The darker gray (red on line) lattice points on the bottom line outside the boundary are missing and need to be supplied by the boundary conditions.

\begin{tabular}{|c|c|}
\hline Moments & Combination of unknowns \\
\hline \hline$\rho, \rho u_{y}, \Pi_{y y}$ & $f_{2}+f_{5}+f_{6}$ \\
\hline$\rho u_{x}, \Pi_{x y}, Q_{x y y}$ & $f_{5}-f_{6}$ \\
\hline$\Pi_{x x}, Q_{x x y}, R_{x x y y}$ & $f_{5}+f_{6}$ \\
\hline \multicolumn{2}{|c|}{ TABLE 1 } \\
\multicolumn{2}{|c|}{ Moment groups at a southern boundary }
\end{tabular}

Expressions for the non-conserved moments must be found by taking moments of the transformation (1.19). For example, the momentum flux tensor is

$$
\boldsymbol{\Pi}=\frac{2 \tau \overline{\boldsymbol{\Pi}}+\Delta t \boldsymbol{\Pi}^{(0)}+\tau \Delta t(\mathbf{F u}+\mathbf{u F})}{2 \tau+\Delta t},
$$

where $\overline{\boldsymbol{\Pi}}=\sum_{i} \bar{f}_{i} \boldsymbol{\xi}_{i} \boldsymbol{\xi}_{i}$. From (1.25) we can find the deviatoric stress $\mathbf{T}=\boldsymbol{\Pi}^{(0)}-\boldsymbol{\Pi}$ in terms of the moments of $\tilde{f}_{i}$ :

$$
\mathbf{T}=\frac{2 \tau\left(\boldsymbol{\Pi}^{(0)}-\bar{\Pi}\right)-\tau \Delta t(\mathbf{F u}+\mathbf{u} \mathbf{F})}{2 \tau+\Delta t} .
$$

2. Boundary conditions. At a straight wall the D2Q9 lattice has three unknown "incoming" (unknown) distributions which need to be supplied by the boundary conditions. To solve the lattice Boltzmann equation one usually imposes boundary conditions directly upon the distributions $f_{i}$. Due to the invertible relationship between the discrete velocity distribution function and its moments, an alternative method would impose constraints on three judiciously chosen moments and then translate these into the particle basis [3]. Since this methods is used in the Sections that follow, it is discussed in some detail here. To illustrate the moment-based apporach, let's consider a horizontal solid wall at a southern boundary. The three incoming (unknown) distribution functions are $f_{2}, f_{5}$ and $f_{6}$, as shown in Figure 2. Table 1 shows how these three unknowns appear in each of the nine moments at the wall $[3,37]$.

The three rows of Table 1 are linearly independent. Therefore we may impose a boundary condition on one moment from each row of Table 1 and solve for the incoming distributions. All other wall moments can be expressed in terms of known $f_{i}$ and the imposed constraints. It is logical to choose the moments that correspond to the hydrodynamic quantities: density, momentum, and momentum flux, rather than the higher-order moments $\mathbf{Q}$ and $\mathbf{R}$. For 
Navier-Stokes flow with no-slip walls, a possible set of constraints would be

$$
\rho u_{x}=\rho u_{y}=0, \quad \Pi_{x x}=\Pi_{x x}^{(0)}=c_{s}^{2} \rho .
$$

The condition on $\Pi_{x x}$ follows from the zero wall velocity constraint and the commonly held assumption that $\Pi_{x x}=\Pi_{x x}^{(0)}+\tau \Pi_{x x}^{(1)}$, where $\Pi_{x x}^{(1)} \propto \partial u_{x} / \partial x$. These are the conditions used in the original moment method [3] and most subsequent work [4, 37, 1, 22, 40, 33]. Note that this is not a condition on the pressure or density. It is a condition on the tangential component of the momentum flux, saying that non-equilibrium parts much vanish at the boundary. The density (and thus pressure) is not imposed; it is computed from known values and dependent moments at the boundary, as shown in equation (2.3), for example.

The second-order discretisation (1.20) requires us to find the unknown (incoming) $\bar{f}_{i}$, rather than $f_{i}$. In the absence of a body force, the conserved moments may be calculated from $\bar{f}_{i}$ in precisely the same way as from $f_{i}$. However, if a source term $S_{i}$ is included, one must be careful to respect equation (1.24). Conditions on the stress must be re-expressed using equation (1.25). Conveniently, the simple Navier-Stokes stress boundary condition becomes $\bar{\Pi}_{x x}=\Pi^{(0)}$. In terms of the incoming distribution functions, the conditions (2.1) are

$$
\begin{aligned}
& \bar{f}_{2}=\bar{f}_{1}+\bar{f}_{3}+\bar{f}_{4}+2\left(\bar{f}_{7}+\bar{f}_{8}\right)-\frac{\rho}{3}, \\
& \bar{f}_{5}=-\bar{f}_{1}-\bar{f}_{8}+\frac{\rho}{6}-\frac{G \rho \Delta t}{4}, \\
& \bar{f}_{6}=-\bar{f}_{3}-\bar{f}_{7}+\frac{\rho}{6}+\frac{G \rho \Delta t}{4},
\end{aligned}
$$

where the wall density is given by

$$
\rho=\bar{f}_{0}+\bar{f}_{1}+\bar{f}_{3}+2\left(\bar{f}_{4}+\bar{f}_{7}+\bar{f}_{8}\right) .
$$

Similar expressions can be found for the incoming $\bar{f}_{i}$ at other boundaries.

We now use the moment method with Navier-Stokes conditions to simulate Poiseuille flow in an infinitely long 2D planar channel (the computational domain is periodic in the streamwise direction). The flow is driven by the force $\mathbf{F}=\left(F_{x}, F_{y}\right)=(\rho G, 0)$, where $G$ is a constant mimicing the pressure gradinet. The relevant non-dimensional number is the Reynolds number, $R e=U_{c} H / \nu$, where $U_{c}=H^{2} G / 8 \nu$ is the centerline velocity. $H=$ $(n-1) \Delta x$ is the channel height and $n$ is the number of grid points in the vertical direction. The spanwise velocity $u_{y}$ and the normal component of the extra stress $T_{y y}$ are zero and the shear stress given by a simple linear profile. This will be proven analytically in Section 3. The density was confirmed to be constant in all cases and the spanwise velocity zero - this is verified theoretically in the Section 3 but previously known from He et al. [25].

Figure 3 plots the non-dimensional streamwise velocity and tangential stress when $R e=$ $100, M a=0.1 \sqrt{3}$ and $n=33$. We emphasise that the numerical solution for $u_{x}$ is exact to floating point round-off error. Moreover, the exact solution is still obtained when using the minimum number of grid points, $n=3$, required to define the characteristic length, $H$, with the moment-method. The plot of tangential stress $T_{x x}$, however, shows large spurious oscillations, as first noticed by Reis [36]. These are generated at the boundary but can infect the flow in the bulk. Doubling the resolution allows us to capture the correct behaviour away from the walls but the spurious oscillations, although smaller in magnitude and rapidly decaying, remain, as shown in Figure 4. Lowering the Mach number by an order of magnitude, on the otherhand, reduces the Knudsen number and thus $\tau$ (note that $K n \propto M a / R e$ ), which emphasises the inconsistency between the Navier-Stokes stress boundary condition and the 

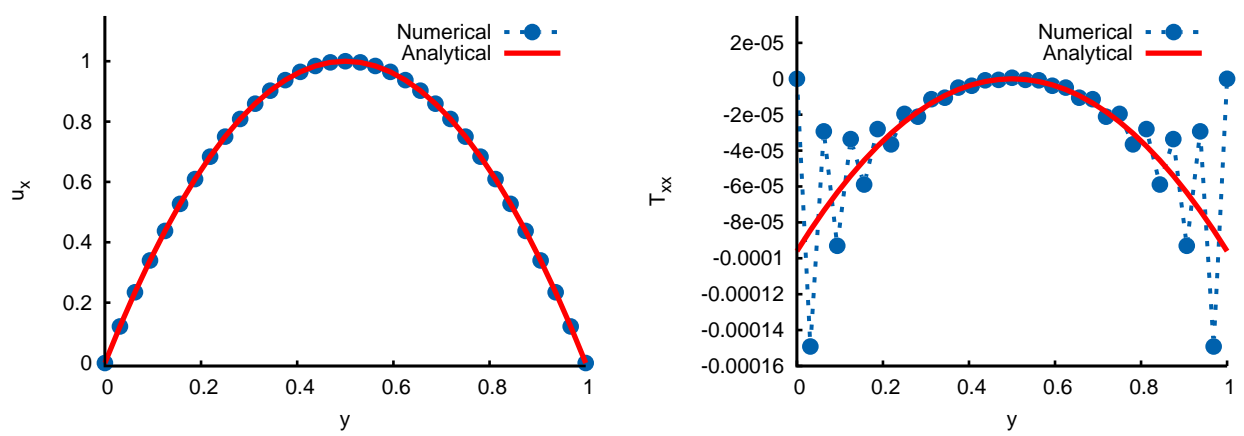

FIG. 3. Plot of the streamwise velocity (left) and tangential stress in an infinitely long two dimensional planar channel flow using the original moment-based boundary conditions (2.2) when $R e=100, M a=0.1 \sqrt{3}$ and $n=33$.

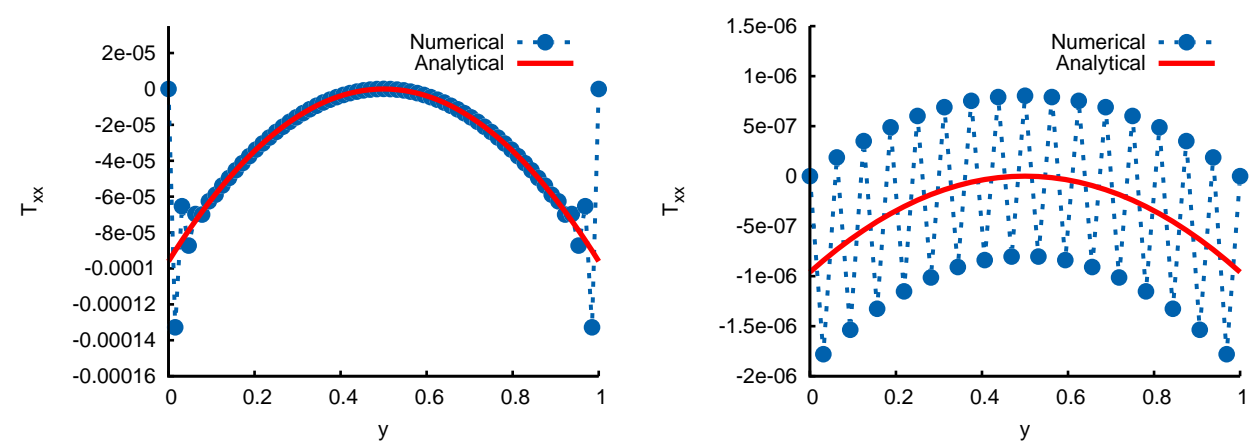

FIG. 4. (colour online) Plot of the analytical and computed solutions of the tangential stress in an infinitely long planar channel flow using Navier-Stokes boundary conditions (2.2) when Re $=100$. Left: $n=65, M a=0.1 \sqrt{3}$. Right: $n=33$ and $M a=0.01 \sqrt{3}$.

lattice Boltzmann equation, as observed in Figure 4. Further explanation will be given in Section 3. The deviatoric stress is approaching the Navier-Stokes solution $\left(T_{x x} \rightarrow 0\right.$ as $K n \rightarrow 0$ ), but the numerical oscillations, although small in magnitude, can infect the entire domain.

Microscale flows typically operate in the small Reynolds number (diffusion dominated) regime. Figure 5 plots the deviatoric stress at $R e=0.1$ when $M a=0.01 \sqrt{3}(K n=$ $\left.\mathcal{O}\left(10^{-1}\right)\right)$ and $M a=0.001 \sqrt{3}\left(K n=\mathcal{O}\left(10^{-2}\right)\right)$. Grid scale oscillations are no longer present, which we will show later is due to a larger value of $\tau$ (c.f. Section 3). However, the computed stress for the $K n=\mathcal{O}\left(10^{-1}\right)$ case is completely incorrect over the whole channel while the $K n=\mathcal{O}\left(10^{-2}\right)$ is correct in the interior but deviates substantially from the analytical solution near the boundaries. We now investigate the LBE computations of the stress in the same flow with some commonly used boundary conditions.

2.1. Bounce-back. The bounce-back method generally places the wall between grid points; its precise location is a function of the kinematic viscosity and, with the BGK collision operator, only asymptotically coincides with the point halfway between nodes. The offset of 

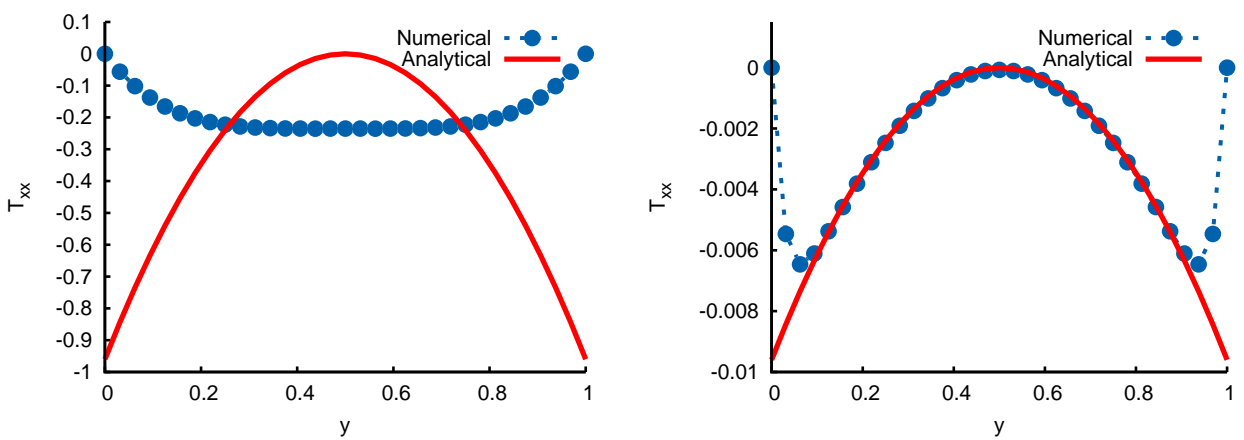

FIG. 5. (colour online) Plot of the analytical and computed solutions of the tangential stress in an infinitely long planar channel flow using Navier-Stokes boundary conditions (2.2)when Re $=0.1$. Left: $n=33, M a=0.01 \sqrt{3}$. Right: $n=33$ and $M a=0.001 \sqrt{3}$.
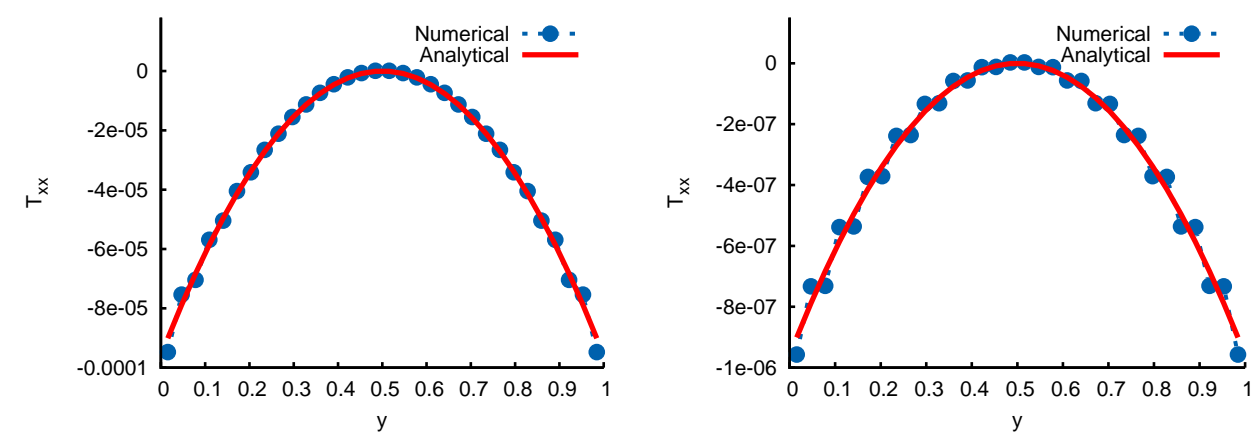

FIG. 6. (colour online) Plot of the analytical and computed solution of the tangential stress in an infinitely long planar channel flow using bounce-back boundary conditions when $R e=100$ and $n=32$. Left: $M a=0.1 \sqrt{3}$. Right: $M a=0.01 \sqrt{3}$.

the wall from the midway point is $\mathcal{O}\left(\Delta x^{2}\right)$ [16]. The bounce-back method is defined by

$$
f_{\bar{i}}(\mathbf{x}, t+\Delta t)=f_{i}^{\star}(\mathbf{x}, t),
$$

where $i$ is the incoming direction and opposite to $\bar{i}\left(\boldsymbol{\xi}_{i}=-\boldsymbol{\xi}_{\bar{i}}\right)$ and $f_{i}^{\star}$ denotes the postcollision distribution function. We note that for a second order in time implementation one should generally transform between $\bar{f}_{i}$ and $f_{i}$ (equation (1.19)) before applying the bounceback rule, but this is not necessary for steady, constant body force, flow. Note also that we do not consider variations to bounce-back that use interpolation techniques to prescribe conditions at specific locations (on or off node), such as Bouzidiet. al. [5].

Figure 6 plots the tangential component of the deviatoric stress using bounce-back boundary conditions with $M a=0.1 \sqrt{3}$ and $M a=0.01 \sqrt{3}$. In both examples $n=32$ and $R e=100$ (this number of grid points is chosen to ensure the same grid spacing as the computations using "on-node" boundary conditions) . Although some oscillations are visible, they are a lot smaller in amplitude than in those with the original moment-based conditions. This is because bounce-back does not explicitly impose an inconsistent condition on the stress 

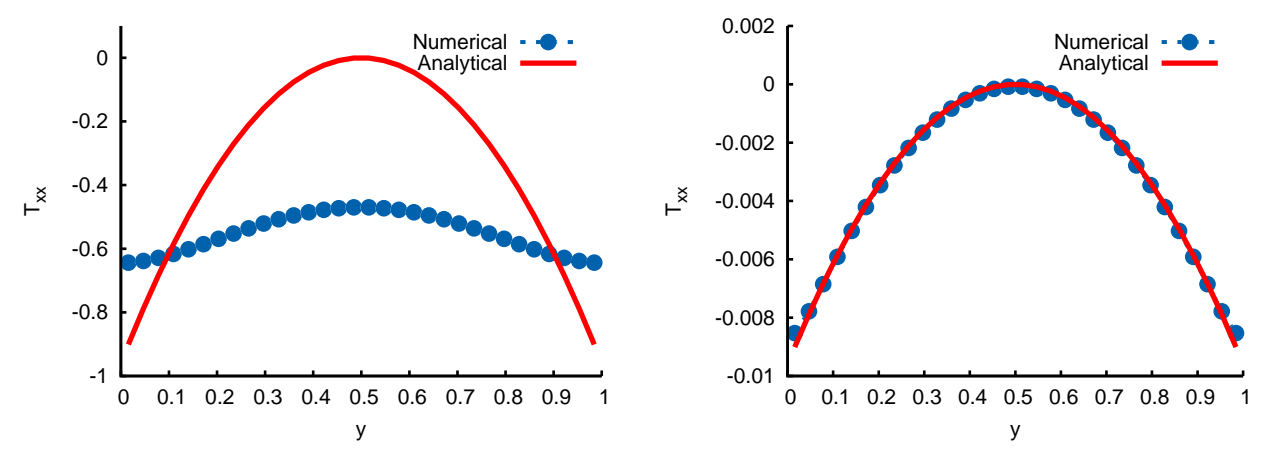

FIG. 7. (colour online) Plot of the analytic and computed solutions of the tangential stress in an infinitely long planar channel flow using bounce-back boundary conditions when Re $=0.1$. Left: $n=32, M a=0.01 \sqrt{3}$. Right: $n=32$ and $M a=0.001 \sqrt{3}$.

Figure 7 plots the deviatoric stress at $R e=0.1$ when $M a=0.01 \sqrt{3}$ and $M a=$ $0.001 \sqrt{3}$. The bounce-back method also predicts qualitatively incorrect behaviour when $M a=\mathcal{O}\left(10^{-1}\right)$. Reducing $M a$ by an order of magnitude predicts the stress correctly in the bulk but deviation from the analytic solution is observed at the boundary. The computations with bounce-back are more accurate than the original moment method because no constraints have been placed on $T_{x x}$ explicitly.

2.2. Non-equilibrium bounce-back. Like the moment method, non-equilibrium bounceback [53] places conditions precisely on grid points. It insists on the exact satisfaction of the no-slip condition $(\mathbf{u}=0)$ at boundary points. To close the system it is assumed that the nonequilibrium part of velocity distribution functions normal to a boundary is "bounced-back". For example, at a south wall it is assumed that $f_{2}-f_{2}^{(0)}=f_{4}-f_{4}^{(0)}$. If a no-slip condition is imposed, the resulting incoming distribution functions at such a wall are found to be

$$
\begin{aligned}
& f_{2}=f_{4}, \\
& f_{5}=f_{7}-\frac{1}{2}\left(f_{1}-f_{3}\right), \\
& f_{6}=f_{8}+\frac{1}{2}\left(f_{1}-f_{3}\right) .
\end{aligned}
$$

The non-equilibrium bounce-back scheme may be given an entirely equivalent interpretation in terms of the velocity moments. The conditions (2.5) can be obtained by imposing the moment constraints [3]

$$
\rho u_{x}=0, \quad \rho u_{y}=0, \quad Q_{x x y}=0 .
$$

The two velocity conditions are useful in defining the problem, but the condition on a component of the third order (non-hydrodynamic) moment seems somewhat arbitrary. In terms of the second order $\left(\bar{f}_{i}\right)$ discretisation, the non-equilibrium bounce-back method becomes

$$
\begin{aligned}
& \bar{f}_{2}=\bar{f}_{4}, \\
& \bar{f}_{5}=\bar{f}_{7}-\frac{1}{2}\left(\bar{f}_{1}-\bar{f}_{3}\right)-\frac{G \rho}{4}, \\
& \bar{f}_{6}=\bar{f}_{8}+\frac{1}{2}\left(\bar{f}_{1}-\bar{f}_{3}\right)+\frac{G \rho}{4} .
\end{aligned}
$$



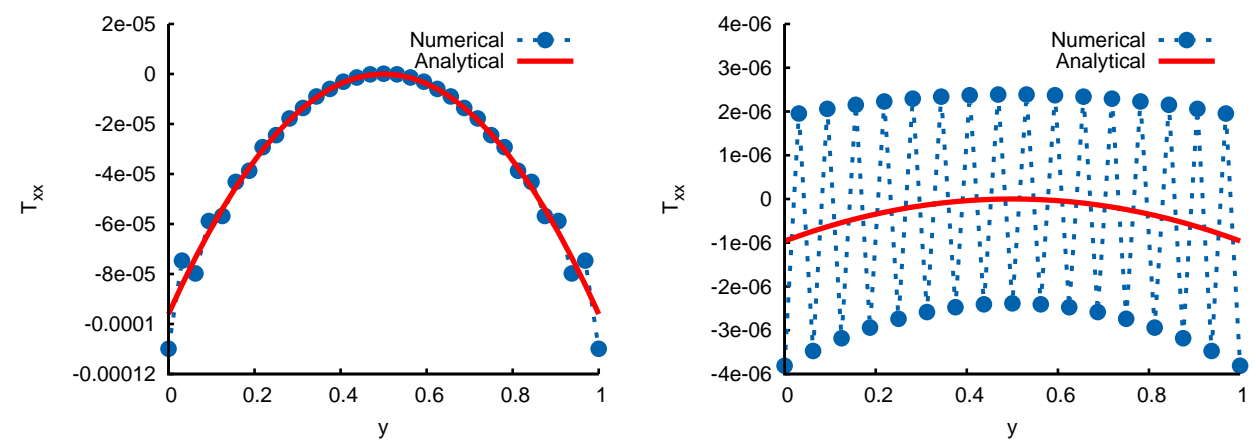

FIG. 8. (colour online) Plot of the analytic and computed solutions of the tangential stress in an infinitely long planar channel flow using non-equilibrium bounce-back boundary conditions when $n=33$ and Re $=100$. Left: $M a=0.1 \sqrt{3}$. Right: $M a=0.01 \sqrt{3}$.
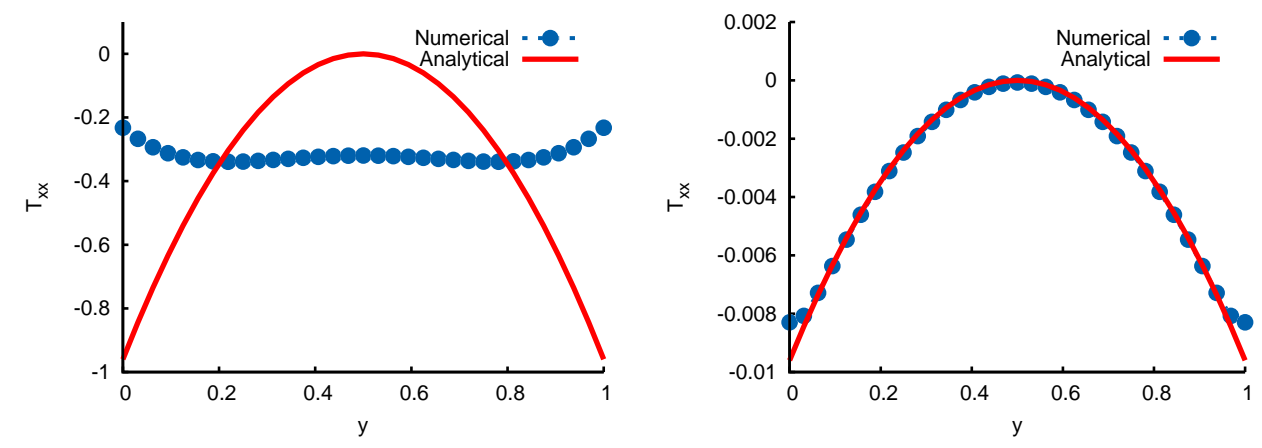

FIG. 9. (colour online) Plot of the analytic and computed solutions of the tangential stress in an infinitely long planar channel flow using non-equilibrium bounce-back boundary conditions when Re $=0.1$. Left: $n=33$, $M a=0.01 \sqrt{3}$. Right: $n=33$ and $M a=0.001 \sqrt{3}$.

Figure 8 plots the tangential component of the deviatoric stress using non-equilibrium bounce-back boundary conditions with $M a=0.1 \sqrt{3}$ and $M a=0.01 \sqrt{3}$. In both examples $n=33$ and $R e=100$. Although we notice the spurious behaviour near the wall with the larger Mach number, they are not as severe as the Navier-Stokes stress boundary conditions. This is because no explicit condition has been imposed on $\Pi_{x x}$. However, when we reduce the Mach number, the oscillations infect the entire flow domain and are larger in magnitude than those observed in Figure 4.

Figure 9 plots the deviatoric stress at $R e=0.1$ when $M a=0.01 \sqrt{3}$ and $M a=$ $0.001 \sqrt{3}$. Non-equilibrium bounce-back is shown to predict behaviour similar to the moment method with Navier-Stokes stress conditions when $M a=\mathcal{O}\left(10^{-1}\right)$. The simulation with the smaller $M a$ fails to predict the correct wall behaviour. We notice the error is smaller than the computations with the moment method, but larger than with bounce-back.

2.3. Diffuse reflection. Maxwell's kinetic boundary conditions for Boltzmann's equation [32] express the incoming distributions as

$$
f(\mathbf{x}, \boldsymbol{\xi}, t)=(1-\alpha) f(\mathbf{x}, \boldsymbol{\xi}-2 \mathbf{n n} \cdot \boldsymbol{\xi}, t)+\alpha f_{w}^{(0)}(\mathbf{x}, \boldsymbol{\xi}, t), \quad \boldsymbol{\xi} \cdot \mathbf{n}>0,
$$



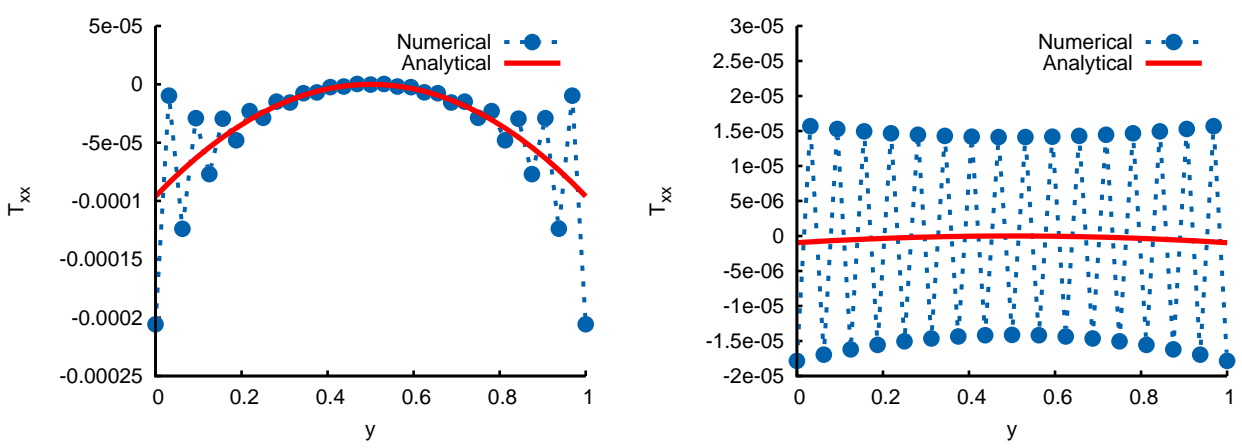

FIG. 10. (colour online) Plot of the analytic and computed solutions of the tangential stress in an infinitely long planar channel flow using Maxwell-Broadwell boundary conditions when $n=33, R e=100$. Left: $M a=0.1 \sqrt{3}$. Right: $M a=0.01 \sqrt{3}$.

where $\alpha$ is the accommodation coefficient and $f_{w}^{(0)}$ is the Maxwell-Boltzmann distribution evaluated at the wall. The first term describes a specular reflection and the second the emission of an $f_{w}^{(0)}$ distribution of particles from the wall. Setting $\alpha=1$ gives the diffuse reflection condition. These conditions were adapted to a finite particle velocity set by Broadwell [6] and analysed in further detail by Gatignol [15]. More recently, Ansumali and Karlin [2] applied this method to the lattice Boltzmann equation. For the D2Q9 velocity set with zero wall velocity, the purely diffusive Maxwell-Broadwell conditions on the south wall are

$$
f_{i}=f_{i}^{(0)} \frac{f_{4}+f_{7}+f_{8}}{f_{2}^{(0)}+f_{5}^{(0)}+f_{6}^{(0)}}, \quad \text { for } i \in\{2,5,6\} .
$$

These are translated into the moment basis as [37]

$$
\rho u_{y}=0, \quad Q_{x x y}=\frac{1}{3} \Pi_{y y}-R_{x x y y}, \quad Q_{x y y}=-\Pi_{x y},
$$

and the Maxwell-Braodwell conditions in terms of the $\bar{f}_{i}$ variables are

$$
\begin{aligned}
& \bar{f}_{2}=\frac{2}{3}\left(\bar{f}_{4}+\bar{f}_{7}+\bar{f}_{8}\right), \\
& \bar{f}_{5}=\frac{1}{6}\left(\bar{f}_{4}+\bar{f}_{7}+\bar{f}_{8}\right)+\frac{\rho G}{24}, \\
& \bar{f}_{6}=\frac{1}{6}\left(\bar{f}_{4}+\bar{f}_{7}+\bar{f}_{8}\right)-\frac{\rho G}{24} .
\end{aligned}
$$

Figure 10 plots the tangential component of the deviatoric stress using Maxwell-Braodwell boundary conditions with $M a=0.1 \sqrt{3}$ and $M a=0.01 \sqrt{3}$. In both examples $n=33$ and $R e=100$. Strong oscillations are once again observed near the walls. The computed stress approximates the analytical solution well in the bulk when the Mach number is large. When $M a$ is reduced (smaller Knudsen number), the magnitude of the stress is smaller but spurious the oscillations infect the entire domain. They are stronger with the Maxwell-Broadwell boundary condition than with the original moment method.

Figure 11 plots the deviatoric stress at $R e=0.1$ when $M a=0.01 / \sqrt{3}$. The purely diffusive Maxwell-Broadwell condition predicts behaviour very similar to bounce-back when $M a=\mathcal{O}\left(10^{-1}\right)$; the only noticeable difference being the location of the wall (recall the 

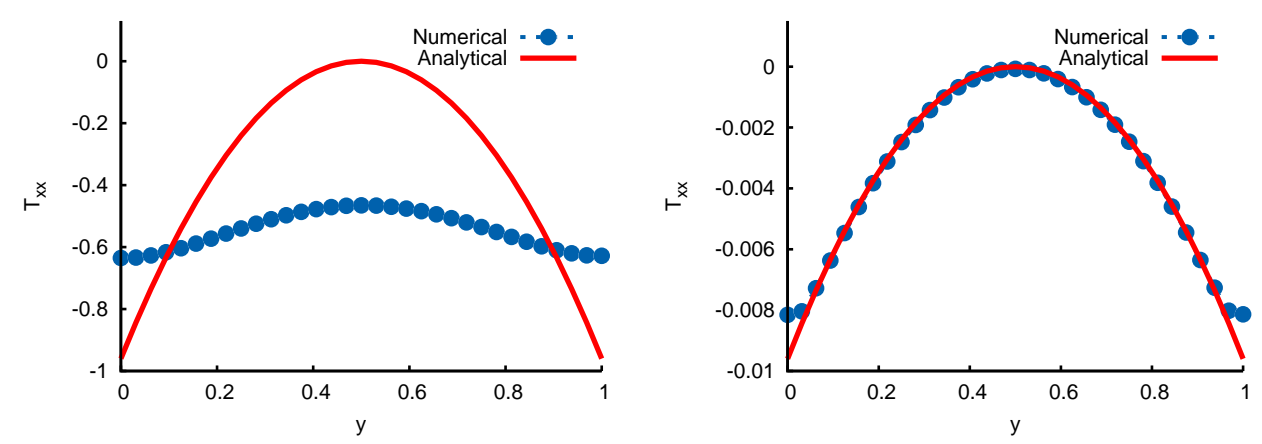

FIG. 11. (colour online) Plot of the analytic and computed solutions of the tangential stress in an infinitely long planar channel flow using non-equilibrium bounce-back boundary conditions when Re $=0.1$. Left: $n=33$, $M a=0.01 \sqrt{3}$. Right: $n=33$ and $M a=0.001 \sqrt{3}$.

boundary is positioned between grid points for bounce-back). Reducing $M a$ by an order of magnitude once again allows for accurate computations of the bulk flow but errors at the wall remain.

3. Discrete solutions for unidirectional planar channel flow. We now follow closely He et al. [25] and find the discrete solution of the lattice Boltzmann equation (1.20) for timeindependent planar channel flow in an infinitely long channel subject to the the boundary conditions (2.1). The flow is driven by a force that mimics the constant pressure gradient, $\mathbf{F}=\left(F_{x}, F_{y}\right)=(\rho G, 0)$, where $G$ is a constant. To ease notation we write the velocity components as $\mathbf{u}=\left(u_{x}, u_{y}\right)=(u, v)$ and work in so-called lattice units with $\Delta x=\Delta t=$ 1. The flow domain is a channel of height $H$ consisting of $n$ computational nodes in the vertical direction with solid walls located at $j=1$ and $j=n$. Since there is no time nor $x$-dependence, the components of equation (1.20) are 


$$
\begin{aligned}
\bar{f}_{0}^{j} & =\frac{4 \rho}{9}\left(1-\frac{3}{2}\left(u_{j}^{2}+v_{j}^{2}\right)\right)-\frac{4 \tau \rho G}{3(\tau+1 / 2)} u_{j}, \\
\bar{f}_{1}^{j} & =\frac{\rho}{9}\left(1+3 u_{j}+3 u_{j}^{2}-\frac{3 v_{j}^{2}}{2}\right)+\frac{\tau \rho G}{3}\left(2 u_{j}+1\right) \\
\bar{f}_{2}^{j} & =\frac{\rho}{9(\tau+1 / 2)}\left(1+3 v_{j-1}+2 v_{j-1}^{2}-\frac{3 u_{j-1}^{2}}{2}\right)-\frac{\tau \rho G}{3(\tau+1 / 2)} u_{j-1}+\frac{\tau-1 / 2}{\tau+1 / 2} \bar{f}_{2}^{j-1}, \\
\bar{f}_{3}^{j} & =\frac{\rho}{9}\left(1-3 u_{j}+3 u_{j}^{2}-\frac{3 v_{j}^{2}}{2}\right)+\frac{\tau \rho G}{3}\left(2 u_{j}-1\right), \\
\bar{f}_{4}^{j} & =\frac{\rho}{9(\tau+1 / 2)}\left(1-3 v_{j+1}+3 v_{j+1}^{2}-\frac{3 u_{j+1}^{2}}{2}\right)-\frac{\tau \rho G}{3(\tau+1 / 2)} u_{j+1}+\frac{\tau-1 / 2}{\tau+1 / 2} \bar{f}_{4}^{j+1}, \\
\bar{f}_{5}^{j} & =\frac{\rho}{36(\tau+1 / 2)}\left(1+3 u_{j-1}+3 v_{j-1}+3 u_{j-1}^{2}+3 v_{j-1}^{2}+9 u_{j-1} v_{j-1}\right) \\
& +\frac{\tau \rho G}{12(\tau+1 / 2)}\left(1+2 u_{j-1}\right)+\frac{\tau-1 / 2}{\tau+1 / 2} \bar{f}_{5}^{j-1}, \\
\bar{f}_{6}^{j} & =\frac{\rho}{36(\tau+1 / 2)}\left(1-3 u_{j-1}+3 v_{j-1}+3 u_{j-1}^{2}+3 v_{j-1}^{2}-9 u_{j-1} v_{j-1}\right) \\
& -\frac{\tau \rho G}{12(\tau+1 / 2)}\left(1-2 u_{j-1}\right)+\frac{\tau-1 / 2}{\tau+1 / 2} \bar{f}_{6}^{j-1}, \\
\bar{f}_{7}^{j} & =\frac{\rho}{36(\tau+1 / 2)}\left(1-3 u_{j+1}-3 v_{j+1}+3 u_{j+1}^{2}+3 v_{j+1}^{2}+9 u_{j+1} v_{j+1}\right) \\
& -\frac{\tau \rho G}{12(\tau+1 / 2)}\left(1-2 u_{j+1}\right)+\frac{\tau-1 / 2}{\tau+1 / 2} \bar{f}_{7}^{j+1}, \\
\bar{f}_{8}^{j} & =\frac{\rho}{36(\tau+1 / 2)}\left(1+3 u_{j+1}-3 v_{j+1}+3 u_{j+1}^{2}+3 v_{j+1}^{2}-9 u_{j+1} v_{j+1}\right) \\
& +\frac{\tau \rho G}{12(\tau+1 / 2)}\left(1+2 u_{j+1}\right)+\frac{\tau-1 / 2}{\tau+1 / 2} \bar{f}_{8}^{j+1} .
\end{aligned}
$$

The notation $\bar{f}_{i}^{j}$ denotes the the distribution function $\bar{f}_{i}$ at node $j$; similarly for $u_{j}$ and $v_{j}$.

At this stage it is worth noting the special case $\tau=1 / 2$. With this choice of relaxation time the recurrence in $\bar{f}_{i}^{j}$ is removed and each distribution function only depends on $u_{j}$ and $v_{j}$. Equivalently, the moments of $\bar{f}_{i}$ at node $j$ only depend on the equilibrium function, $f_{i}^{(0)}$, at $j$ and at its nearest neighbours. This specific choice corresponds to the lattice kinetic scheme of Inamuro [28].

As in He et al. [25], The velocity in the bulk, $2 \leq j \leq n-1$, is found according to the first order moment of $\bar{f}_{i}$,

$$
\sum_{i} \bar{f}_{i}^{j} \xi_{\alpha}=\rho \bar{u}_{j}=\rho u_{j}-\frac{1}{2} \sum_{i} S_{i}^{j}=\left(\bar{f}_{1}^{j}-\bar{f}_{3}^{j}+\bar{f}_{5}^{j}-\bar{f}_{6}^{j}+\bar{f}_{8}^{j}-\bar{f}_{7}^{j}\right)
$$


which, upon using the above recurrence relation for $\bar{f}_{i}$, becomes

$$
\begin{aligned}
\rho u_{j}-\frac{\rho G}{2} & =\frac{2 u_{j}}{3}+\frac{2 \tau \rho G}{3}+\frac{1}{\tau+1 / 2}\left(\frac{u_{j-1}}{6}+\frac{u_{j-1} v_{j-1}}{2}\right) \\
& +\frac{1}{\tau+1 / 2}\left(\frac{u_{j+1}}{6}-\frac{u_{j+1} v_{j+1}}{2}\right)+\frac{\tau \rho G}{3(\tau+1 / 2)} \\
& +\frac{\tau-1 / 2}{\tau+1 / 2}\left(\bar{f}_{5}^{j-1}-\bar{f}_{6}^{j-1}+\bar{f}_{8}^{j+1}-\bar{f}_{7}^{j-1}\right) .
\end{aligned}
$$

We use the recurrence relations for $\bar{f}_{i}^{j}$ to find expressions for $\left(\bar{f}_{5}^{j-1}-\bar{f}_{6}^{j-1}\right)$ and $\left(\bar{f}_{8}^{j+1}-\bar{f}_{7}^{j+1}\right)$ :

$$
\begin{aligned}
& \left(\bar{f}_{5}^{j-1}-\bar{f}_{6}^{j-1}\right)=\rho u_{j-1}-\frac{\rho G}{2}-\left(\bar{f}_{1}^{j-1}-\bar{f}_{3}^{j-1}\right)-\left(\bar{f}_{8}^{j-1}-\bar{f}_{7}^{j-1}\right) \\
& \left(\bar{f}_{8}^{j+1}-\bar{f}_{7}^{j+1}\right)=\rho u_{j+1}-\frac{\rho G}{2}-\left(\bar{f}_{1}^{j+1}-\bar{f}_{3}^{j=1}\right)-\left(\bar{f}_{5}^{j+1}-\bar{f}_{6}^{j+1}\right) .
\end{aligned}
$$

Substituting these into (3.2) and using the recurrence again to eliminate the $\bar{f}_{i}^{j \pm 1}$ in favour of $\bar{f}_{i}^{j}$ yields

$$
\frac{u_{j+1} v_{j+1}-u_{j-1} v_{j-1}}{2}=\nu\left(u_{j+1}+u_{j-1}-2 u_{j}\right)+G,
$$

and we remind the reader that $\nu=\tau / 3$. Equation (3.6) is the second order finite-difference form of the incompressible Navier-Stokes equations with a constant body force:

$$
\frac{\partial(u v)}{\partial y}=\nu \frac{\partial^{2} u}{\partial y^{2}}+G
$$

The recurrence relations for $\bar{f}_{i}$ show also (as in [25], likewise) that

$$
v_{j+1}^{2}-v_{j-1}^{2}=2 \tau\left(v_{j+1}-2 v_{j}+v_{j-1}\right)
$$

and

$$
v_{j+1}^{2}-2 v_{j}^{2}+v_{j-1}^{2}=-2 \tau\left(v_{j+1}-v_{j-1}\right) .
$$

Adding and subtracting these two equations tells us that $v_{j}$ must be independent of $j$. Since the boundaries impose $v_{1}=v_{n}=0$, we conclude that $v_{j}=0$ for all $j$. The equation (3.7) with $v_{j}=0$ is a linear ordinary difference equation for $u_{j}$ with solution

$$
u_{j}=\frac{4 U_{c}}{(n-1)^{2}}(j-1)(n-j)+U_{s}, \quad j=1,2, \ldots n
$$

where $U_{c}=H^{2} G / 8 \nu$ is the center-line velocity and $H=(n-1)$ is the channel height. $U_{s}$ is the "numerical slip" which depends on the boundary conditions. It is a constant so the solution, regardless of the boundary conditions, is a perfect parabola off-set from zero at the boundaries by an amount $U_{s}$. Thus there are no boundary layers in the velocity for the D2Q9 LBE. For the moment method, $u_{1}=u_{n}=0$, as imposed by equation (2.1). Therefore $U_{s}=0$ and the exact solution for the velocity in Poiseuille flow is obtained. 
3.1. The stress tensor. The recurrence relations for $\bar{f}_{i}$ can also be solved for the three components of the stress tensor. Starting with the normal component of the momentum flux,

$$
\begin{aligned}
\bar{\Pi}_{y y}^{j} & =\bar{f}_{2}^{j}+\bar{f}_{4}^{j}+\bar{f}_{5}^{j}+\bar{f}_{6}^{j}+\bar{f}_{7}^{j}+\bar{f}_{8}^{j} \\
& =\frac{2 \rho}{3(2 \tau+1)}+\frac{2 \tau-1}{2 \tau+1}\left(\bar{f}_{2}^{j-1}+\bar{f}_{5}^{j-1}+\bar{f}_{6}^{j-1}+\bar{f}_{4}^{j+1}+\bar{f}_{7}^{j+1}+\bar{f}_{8}^{j+1}\right) .
\end{aligned}
$$

We can eliminate the distributions at nodes neighbouring node $j$ by using the recurrence relations for $\bar{f}_{i}$, which tell us that

$$
\begin{aligned}
& \bar{f}_{4}^{j+1}+\bar{f}_{7}^{j+1}+\bar{f}_{8}^{j+1}=-\rho \bar{v}_{j+1}+\bar{f}_{2}^{j+1}+\bar{f}_{5}^{j+1}+\bar{f}_{6}^{j+1}, \\
& \bar{f}_{2}^{j-1}+\bar{f}_{5}^{j-1}+\bar{f}_{6}^{j-1}=\rho \bar{v}_{j-1}+\bar{f}_{4}^{j-1}+\bar{f}_{7}^{j-1}+\bar{f}_{8}^{j-1} .
\end{aligned}
$$

Upon using the recurrence relations again and the fact that $\bar{v}_{j}=0$ we see that $\Pi_{y y}^{j}=\rho / 3$ and thus $T_{y y}^{j}=0$.

For the shear momentum flux,

$$
\begin{aligned}
\bar{\Pi}_{x y}^{j} & =\bar{f}_{5}^{j}-\bar{f}_{6}^{j}+\bar{f}_{7}^{j}-\bar{f}_{8}^{j}, \\
& =\frac{\rho}{3(2 \tau+1)}\left(u_{j-1}-u_{j+1}\right) \\
& +\frac{2 \tau-1}{2 \tau+1}\left(\bar{f}_{5}^{j-1}-\bar{f}_{6}^{j-1}+\bar{f}_{7}^{j+1}-\bar{f}_{8}^{j+1}\right) .
\end{aligned}
$$

We follow the same procedure as above and use equations (3.3) and (3.4) to eliminate $\bar{f}_{5}^{j-1}$ $\bar{f}_{6}^{j-1}+\bar{f}_{7}^{j+1}-\bar{f}_{8}^{j+1}$, and then use the recurrence relations for $\bar{f}_{i}$ again to replace all $\bar{f}_{i}$ evaluated at nodes neighboring node $j$. This gives

$$
\bar{\Pi}_{x y}^{j}=\frac{2 \rho \tau}{3(\tau+1 / 2)}\left(\bar{u}_{j-1}-\bar{u}_{j+1}\right)+\left(\frac{\tau-1 / 2}{\tau+1 / 2}\right)^{2} \bar{\Pi}_{x y}^{j},
$$

which rearranges into

$$
\Pi_{x y}^{j}=-\frac{\rho}{6}(\tau+1 / 2)\left(u_{j+1}-u_{j-1}\right) .
$$

Since $\Pi_{x y}^{(0)}=\rho u v=0$ for unidirectional channel flow, we obtain the exact expression for the shear stress,

$$
T_{x y}=\frac{\rho \tau}{6}\left(u_{j+1}-u_{j-1}\right) .
$$

This is a consistent second order central finite difference approximation to the Navier-Stokes shear stress $T_{x y}=\mu u^{\prime}$.

To find the solution for the tangential stress

$$
\begin{aligned}
\bar{\Pi}_{x x}^{j} & =\left(\bar{f}_{1}^{j}+\bar{f}_{3}^{j}+\bar{f}_{5}^{j}+\bar{f}_{6}^{j}+\bar{f}_{7}^{j}+\bar{f}_{8}^{j}\right), \\
& =\frac{2 \rho}{9}+\frac{\rho}{9(\tau+1 / 2)}+\frac{2}{3} \rho u_{j}^{2}+\frac{\rho}{6(\tau+1 / 2)}\left(u_{j-1}^{2}+u_{j+1}^{2}\right) \\
& +\frac{\tau-1 / 2}{\tau+1 / 2}\left(\bar{f}_{5}^{j-1}+\bar{f}_{6}^{j-1}+\bar{f}_{7}^{j+1}+\bar{f}_{8}^{j+1}\right) \\
& +\frac{4 \tau}{3} \rho G u_{j}+\frac{\tau}{3(\tau+1 / 2)} \rho G\left(u_{j+1}+u_{j-1}\right)
\end{aligned}
$$


we must note that

$$
\begin{aligned}
& \left(\bar{f}_{5}^{j-1}+\bar{f}_{6}^{j-1}\right)=\bar{\Pi}_{x x}^{j-1}-\left(\bar{f}_{1}^{j-1}+\bar{f}_{3}^{j-1}+\bar{f}_{7}^{j-1}+\bar{f}_{8}^{j-1}\right) \\
& \left(\bar{f}_{7}^{j+1}+\bar{f}_{8}^{j+1}\right)=\bar{\Pi}_{x x}^{j+1}-\left(\bar{f}_{1}^{j+1}+\bar{f}_{3}^{j+1}+\bar{f}_{5}^{j+1}+\bar{f}_{6}^{j+1}\right) .
\end{aligned}
$$

Upon using the recurrence relations for $\bar{f}_{i}$ and respecting the transformation (1.26) we find the discrete solution for the tangential stress $T_{x x}$ :

$$
\begin{aligned}
3\left(4 \tau^{2}-1\right)\left(T_{x x}^{j+1}-2 T_{x x}^{j}+T_{x x}^{j-1}\right)-12 T_{x x}^{j} & =4 \tau^{2} \rho\left(u_{j-1}^{2}-2 u_{j}^{2}+u_{j+1}^{2}\right) \\
& -16 \tau^{3} \rho G\left(u_{j+1}+u_{j-1}-2 u_{j}\right) \\
& +6 \tau \rho G\left(u_{j+1}+u_{j-1}+2 u_{j}\right) .
\end{aligned}
$$

We seek a quadratic particular solution of the form $T^{j(P I)}=\alpha j^{2}+\beta j+\gamma$. Substituting into equation (3.19) and using the solution (3.10) for $u_{j}$ with $U_{s}=0$ we find

$$
\begin{aligned}
T_{x x}^{j(P I)} & =\rho G^{2}\left(-6 j^{2}+6 j(n+1)-3 n-\frac{3}{2} n^{2}-16 \tau^{2}+\frac{3}{2}\right) \\
& =-2 \mu \tau\left(\frac{u_{j+1}-u_{j-1}}{2}\right)^{2}+\rho G^{2}\left(16 \tau^{2}-3\right),
\end{aligned}
$$

The first term on the right-hand-side is a second order centered finite difference approximation to $-2 \mu \tau\left(u^{\prime}\right)^{2}$ and the second is an error due to the discretisation of the body force. Since $G \propto c_{s} \Delta t$, its presence is consistent with the second order accuracy of the LBE. This term vanishes when $\tau=\sqrt{3} / 4$.

The homogeneous solution to equation (3.19) is

$$
T_{x x}^{j(h o m)}=A m^{j}+B m^{-j},
$$

where $A$ and $B$ are constants and

$$
m=\frac{2 \tau+1}{2 \tau-1}
$$

We can clearly see the inadequacies of the Navier-Stokes boundary condition for the tangential stress, which explicitly sets $T_{x x}=0$, requiring $A$ and $B$ to be non-zero. We can find the coefficients $A$ and $B$ in this case by using the solution (3.10) for the velocity and the boundary conditions $T_{x x}^{1}=T_{x x}^{n}=0$. To exploit the symmetry of the solution we write $T_{x x}^{j(h o m)}=C m^{j}+D m^{n+1-j}$ so that the boundary conditions $T_{x x}^{1}=T_{x x}^{n}=0$ determine

$$
C=D=-\frac{T^{W}}{m^{n}+m},
$$

where $T^{W}=T_{x x}^{1(P I)}=T_{x x}^{n(P I)}$ is the particular solution (3.20) evaluated at the wall. The complete solution is thus

$$
\begin{aligned}
T_{x x}^{j} & =T_{x x}^{j(h o m)}+T_{x x}^{j(P I)} \\
(3.24) & =-\frac{T^{W}}{m^{n}+m}\left(m^{j}+m^{n+1-j}\right)-2 \mu \tau\left(\frac{u_{j+1}-u_{j-1}}{2}\right)^{2}-\rho G^{2}\left(16 \tau^{2}-3\right) .
\end{aligned}
$$




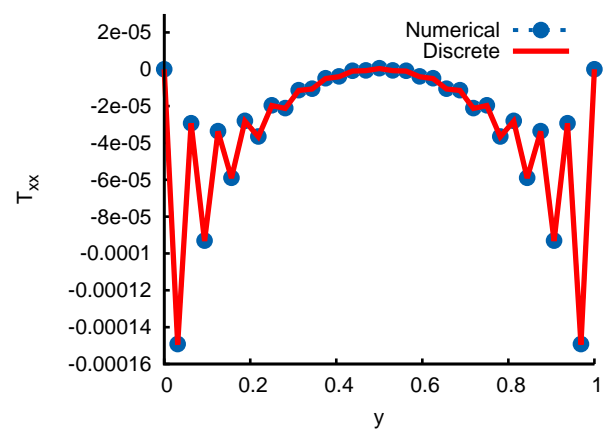

FIG. 12. (colour online) Plot of the discrete ((analytical solution of the LBE (3.24) and computed LBE solutions of the tangential stress in an infinitely long $2 D$ planar channel using the Navier-Stokes moment-based boundary conditions for the $\operatorname{LBE}(2.2)$ when $n=33$ and $M a=0.1 \sqrt{3}$, and $R e=100$.

When $\tau<1 / 2, m^{j}$ changes sign for odd and even $j$. This causes the solution (3.24) to oscillate near the boundary. This inconsistency becomes more severe as $\tau \rightarrow 0$ and $m \rightarrow-1$, and is worse when the number of gridpoints, $n$, is even (because in this case the term in the denominator $m^{n}+m \rightarrow 0$ ). For a fixed Reynolds number, $\tau$ decreases as either $\Delta x$ increases (fewer grid points) or $\Delta t$ decreases (smaller Mach number). This is in agreement with the results reported in Section 2. The discrete solution (3.24) is plotted together with the lattice Boltzmann BGK computation of the tangential component of the deviatoric stress in Figure 12.

3.2. Interpretation of the recurrence relation. The left hand side of (3.19) defines a tridiagonal matrix and reveals the numerical scheme used by the lattice Boltzmann method for the deviatoric stress. The special case of $\tau^{2}=1 / 6$ corresponds to the classic fourth order compact finite difference approximation for second order derivatives at grid points [30]. This value gives precisely the "optimal" collision time reported by Holdych et al. [26] and is similar to that of Zhao [51], as found from a truncation error analysis of the lattice Boltzmann equation. The choice of $\tau^{2}=3 / 16$ will eliminate the body force error while $\tau^{2}=1 / 4 \mathrm{elim}$ inates the recurrence for $T_{x x}$ on the left hand side of equation (3.19). More generally, when $\tau^{2}=1 / 4$ the moments of $\bar{f}_{i}$ only depend on the equilibrium function at nearest neighbours. This corresponds to the lattice kinetic scheme of Inamuro [28]. The above analysis offers further interpretation of the lattice Boltzmann equation in terms of finite difference stencils. Unfortunately, one does not get much freedom to choose numerically favourable values of $\tau$ with the BGK model, at least not without excessive resolution, because $\tau$ is usually set by the viscosity or Reynolds number. The two-relaxation-time collision operator offers a way forward since parameter that controls the effective stencil of the LBE is the product of two different relaxation times, rather than the $\tau^{2}$, as will be discussed in Section 5 .

4. Stress boundary conditions. The previous sections show that the D2Q9 lattice Boltzmann equation has $\mathcal{O}\left(\tau^{2}\right)$ contributions to the stress. Thus the model should be accompanied with compatible boundary conditions for $\boldsymbol{T}$. We follow Reis [36] and seek a wall stress condition that is consistent with (1.16). As before, we illustrate the procedure with a solid wall at the south of the domain, but the method may be applied to all boundaries aligned with grid points.

For completeness, we write the three components of (1.15) for the deviatoric stress at a 
no-slip wall in planar channel flow:

$$
\begin{aligned}
T_{x x}+2 \tau T_{x y} \frac{\partial u_{x}}{\partial y} & =0, \\
T_{y y} & =0, \\
T_{x y}-\mu \frac{\partial u_{x}}{\partial y} & =0 .
\end{aligned}
$$

Substituting equation (4.3) into equation (4.1) gives the required boundary condition for the tangential component of the deviatoric stress,

$$
T_{x x}=-\frac{2 \tau}{\mu} T_{x y}^{2} .
$$

To impose the stress boundary condition upon the lattice Boltzmann equation (1.20) we must enforce constraint (4.4) on $\bar{\Pi}_{x x}$ and then translate this into conditions for the incoming $\bar{f}_{i} . \boldsymbol{T}$ is related to $\bar{\Pi}$ through equation (1.26), and since $u_{x}=0$ on the boundary,

$$
\begin{aligned}
& \bar{\Pi}_{x x}=\frac{\rho}{3}-\frac{2 \tau+1}{2 \tau} T_{x x}, \\
& \bar{\Pi}_{x y}=-\frac{2 \tau+1}{2 \tau} T_{x y} .
\end{aligned}
$$

The above, together with equation (4.4), defines the boundary condition for $\bar{\Pi}_{x x}$ in terms of $\bar{\Pi}_{x y}$ :

$$
\bar{\Pi}_{x x}=\frac{\rho}{3}+\frac{12 \tau}{\rho(2 \tau+1)} \bar{\Pi}_{x y}^{2} .
$$

We can express the wall shear stress in terms of the known distribution functions at the wall:

$$
\bar{\Pi}_{x y}=-\frac{\rho G}{2}-\bar{f}_{1}+\bar{f}_{3}+2 \bar{f}_{7}-2 \bar{f}_{8} .
$$

The appearance of the force $\rho G$ is due to streamwise momentum moment, equation (1.24). Together with the no-slip condition, the incoming $\bar{f}_{i}$ at the south boundary are

$$
\begin{aligned}
& \bar{f}_{2}=\bar{f}_{1}+\bar{f}_{3}+\bar{f}_{4}+2\left(\bar{f}_{7}+\bar{f}_{8}\right)-\frac{\rho}{3}-\frac{12 \tau}{\rho(2 \tau+1)} \bar{\Pi}_{x y}^{2}, \\
& \bar{f}_{5}=-\bar{f}_{1}-\bar{f}_{8}+\frac{\rho}{6}+\frac{6 \tau}{\rho(2 \tau+1)} \bar{\Pi}_{x y}^{2}-\frac{\rho G}{4} \\
& \bar{f}_{6}=-\bar{f}_{3}-\bar{f}_{7}+\frac{\rho}{6}+\frac{6 \tau}{\rho(2 \tau+1)} \bar{\Pi}_{x y}^{2}+\frac{\rho G}{4} .
\end{aligned}
$$

Equivalent expressions are obtained for the unknown $\bar{f}_{i}$ at the north wall. More generally, the boundary condition for the tangential component of the stress is given in terms of the shear stress. If one imposes boundary conditions on the velocity then the wall shear stress can also be formulated in terms of the tangential velocity moment and known (outgoing) distribution functions.

This local method is based on the PDE solutions $(4.1,4.2,4.3)$ of the deviatoric stress at the boundaries but the lattice Boltzmann solution for the stress includes a small error term due the the discretisation of the body force. We can find the analytical solution of the lattice Boltzmann stress with the Burnett boundary conditions by writing

$$
T_{x x}^{j}=\rho G^{2}\left(-6 j^{2}+6 j(n+1)-3 n-\frac{3}{2} n^{2}-16 \tau^{2}+\frac{3}{2}\right)+k\left(m^{j}+m^{n+1-j}\right)
$$



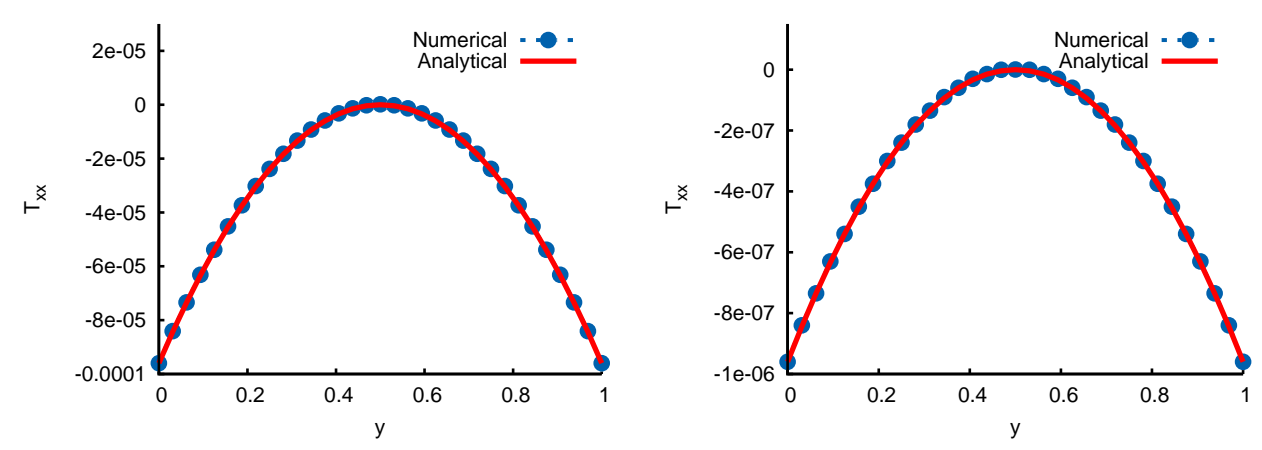

FIG. 13. (colour online) Plot of the discrete analytical solution (4.11) and LBE numerical prediction of the tangential stress in an infinitely long planar channel using the moment-based stress boundary conditions (4.9) when $n=33$ and and $R e=100$. Left: $M a=0.1 \sqrt{3}$. Right: $M a=0.01 \sqrt{3}$.

where the constant, $k$, that multiplies the homogeneous solution is determined by making the error vanish at $j=1$ and $j=n$. Thus the analytical solution of the lattice Boltzmann deviatoric stress with consistent Burnett boundary conditions is

$$
T_{x x}^{j}=-2 \mu \tau\left(\frac{u_{j+1}-u_{j-1}}{2}\right)^{2}+\rho G^{2}\left(16 \tau^{2}-3\right)\left(1-\frac{m^{j}+m^{n+1-j}}{m+m^{n}}\right) .
$$

Equation (4.11) agreed with the computed solutions of the stress to machine precision for all tested parameter values and resolutions. Thus we show instead comparisons with the analytical solution of the partial differential equation for the deviatoric stress. Figure 13 plots the computed and analytical solution of the tangential stress, $T_{x x}$, when $R e=100$ and $n=33$ using the Burnett boundary condition. The oscillations are no longer visible and an excellent agreement between the numerical and exact solution of the PDE observed. The small error at each grid point is precisely the $\rho G^{2}\left(16 \tau^{2}-3\right)\left(1-\left(m^{j}+m^{n+1-j}\right) /\left(m+m^{n}\right)\right)$ contribution to the discrete $T_{x x}^{j}$. For completeness we include a plot of the same flow with $M a=0.01 \sqrt{3}$.

Figure 14 plots the deviatoric stress at $R e=0.1$ when $M a=0.01 \sqrt{3}$ and $M a=$ $0.001 \sqrt{3}$ using the proposed stress boundary condition. The method still predicts completely different behaviour to the solution (1.16) when $M a=\mathcal{O}\left(10^{-1}\right)$. This is due to the slow relaxation of the third order moment $\mathbf{Q}$, which violates the assumptions made in Section 3.1 when deriving the constitutive equation for $T_{\alpha \beta}$. However, the wall behaviour is now predicted precisely and the numerical solutions are free from oscillations. Reducing $M a$ (equivalently, $\Delta t$ ) by an order of magnitude allows us to compute the stress very accurately throughout the channel; something which all boundary conditions discussed in Section 2 failed to do.

5. Two relaxation time models. The two-relaxation-time (TRT) discrete Boltzmann equation relaxes the odd and even order moments at different rates and can be written concisely as

$$
\frac{\partial f_{i}}{\partial t}+\boldsymbol{\xi} \cdot \nabla f_{i}=-\frac{1}{\tau^{+}}\left[\frac{1}{2}\left(f_{i}+f_{\bar{i}}\right)-f_{i}^{(0+)}\right]-\frac{1}{\tau^{-}}\left[\frac{1}{2}\left(f_{i}-f_{\bar{i}}\right)-f_{i}^{(0-)}\right]
$$



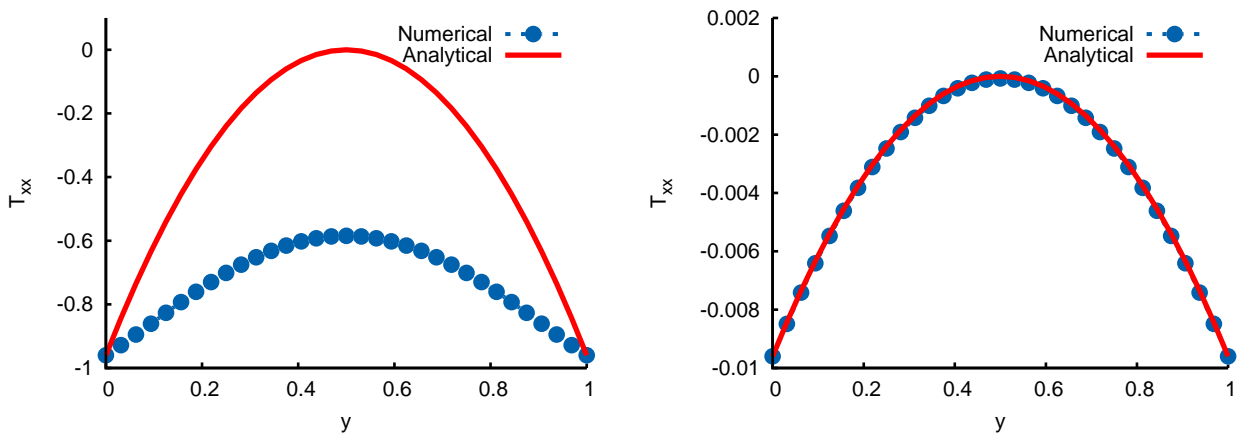

FIG. 14. (colour online) Plot of the discrete analytical solution (4.11) and LBE numerical prediction of the tangential stress in an infinitely long planar channel using the moment-based stress boundary conditions (4.9) when $R e=0.1$. Left: $n=33, M a=0.01 \sqrt{3}$. Right: $n=33$ and $M a=0.001 \sqrt{3}$.

where $\tau^{+}$and $\tau^{-}$are the relaxation times for the even and odd order moments, respectively, $\bar{i}$ is defined by $\boldsymbol{\xi}_{\bar{i}}=-\boldsymbol{\xi}_{i}$, and $f_{i}^{(0-)}$ are the even and odd parts of $f_{i}^{(0)}$ :

$$
\begin{aligned}
f_{i}^{(0+)} & =\rho w_{i}\left(1+\frac{9}{2}\left(\boldsymbol{\xi}_{i} \cdot \mathbf{u}\right)^{2}-\frac{3}{2} \mathbf{u}^{2}\right), \\
f_{i}^{(0-)} & =3 \rho w_{i} \boldsymbol{\xi}_{i} \cdot \mathbf{u} .
\end{aligned}
$$

The BGK equation is recovered when $\tau^{+}=\tau^{-}=\tau$. The zeroth, first and second order moments of (5.1) yield the partial differential equations (1.10)-(1.12), with $\tau$ replaced by $\tau^{+}$. If we do not assume that $\mathcal{Q}_{\alpha \beta \gamma} \approx \mathcal{Q}_{\alpha \beta \gamma}^{(0)}$, as was done in Section 1.1, then the deviatoric stress take the form

$$
T_{\alpha \beta}+\tau^{+}\left[\partial_{t} T_{\alpha \beta}+u_{\gamma} \partial_{\gamma} T_{\alpha \beta}+T_{\alpha \gamma} \frac{\partial u_{\beta}}{\partial \gamma}+T_{\beta \gamma} \frac{\partial u_{\alpha}}{\partial \gamma}-\partial_{\gamma} \mathcal{Q}_{\alpha \beta \gamma}\right]=\tau^{+} \theta \rho\left(\frac{\partial u_{\alpha}}{\partial \beta}+\frac{\partial u_{\beta}}{\partial \alpha}\right)
$$

where $\mathcal{Q}_{\alpha \beta \gamma}$ is the third order moment with respect to the peculiar velocity.

The third-order moment PDE is

$$
\frac{\partial \mathbf{Q}}{\partial t}+\nabla \cdot \mathbf{R}=-\frac{1}{\tau^{-}}\left(\mathbf{Q}-\mathbf{Q}^{0}\right) .
$$

In terms of the peculiar velocity the left hand side of (5.5) becomes

$$
\begin{aligned}
\partial_{t} Q_{\alpha \beta \gamma}+\partial_{\delta} R_{\alpha \beta \gamma \delta} & =\partial_{t}\left(\mathcal{Q}_{\alpha \beta \gamma}+u_{\alpha}\left(c_{s}^{2} \rho \delta_{\beta \gamma}-T_{\beta \gamma}\right)+u_{\beta}\left(c_{s}^{2} \rho \delta_{\gamma \alpha}-T_{\gamma \alpha}\right)\right. \\
& \left.+u_{\gamma}\left(c_{s}^{2} \rho \delta_{\alpha \beta}-T_{\alpha \beta}\right)+\rho u_{\alpha} u_{\beta} u_{\gamma}\right) \\
& +\partial_{\delta}\left[\mathcal{R}_{\alpha \beta \gamma \delta}+u_{\alpha} \mathcal{Q}_{\beta \gamma \delta}+u_{\beta} \mathcal{Q}_{\alpha \gamma \delta}+u_{\gamma} \mathcal{Q}_{\alpha \beta \delta}+u_{\delta} \mathcal{Q}_{\alpha \beta \gamma}\right. \\
& +u_{\alpha} u_{\beta}\left(c_{s}^{2} \rho \delta_{\gamma \delta}-T_{\gamma \delta}\right)+u_{\alpha} u_{\gamma}\left(c_{s}^{2} \rho \delta_{\beta \delta}-T_{\beta \delta}\right) \\
& +u_{\alpha} u_{\delta}\left(c_{s}^{2} \rho \delta_{\beta \gamma}-T_{\beta \gamma}\right)+u_{\beta} u_{\gamma}\left(c_{s}^{2} \rho \delta_{\alpha \delta}-T_{\alpha \delta}\right) \\
& +u_{\beta} u \delta\left(c_{s}^{2} \rho \delta_{\alpha \delta}-T_{\alpha \delta}\right)+u_{\gamma} u_{\delta}\left(c_{s}^{2} \rho \delta_{\alpha \beta}-T_{\alpha \beta}\right) \\
& \left.+\rho u_{\alpha} u_{\beta} u_{\gamma} u_{\delta}\right]
\end{aligned}
$$


where $\mathcal{R}_{\alpha \beta \gamma \delta}=\sum_{i} f_{i} c_{i \alpha} c_{i \beta} c_{i \gamma} c_{i \delta}$.

We proceed by considering Poiseuille flow where, in terms of the peculiar velocity, equations (1.12) and (5.7) reduce to

$$
\begin{aligned}
-2 u^{\prime} T_{x y}+\mathcal{Q}_{x x y}^{\prime} & =\frac{1}{\tau^{+}} T_{x x}, \\
\mathcal{R}_{x x y y}^{\prime} & =-\frac{1}{\tau^{-}}\left(\mathcal{Q}_{x x y}-2 u T_{x y}\right) .
\end{aligned}
$$

The expression for $T_{x y}$ remains unchanged and is given in (1.16). If $\tau^{-} \gg \tau^{+}$, as is likely to be the case for a numerically favourable algorithm (c.f Section 5.1) we cannot assume $\mathcal{Q}_{x x y} \approx \mathcal{Q}^{(0)}$ and must instead seek a more general solution to (5.9). This equation involves the fourth-order tensor $\mathcal{R}_{x x y y}$ which, for planar channel flow, evolves according to

$$
-2 u_{x}^{\prime} T_{x y}+\mathcal{Q}_{x x y}^{\prime}=-\frac{1}{\tau^{+}}\left(\mathcal{R}_{x x y y}-R_{x x y y}^{(0)}\right) .
$$

If we now assume

$$
\mathcal{R}^{\prime} \approx R_{x x y y}^{(0) \prime}=\frac{2}{3} u u^{\prime},
$$

then equation (5.9) becomes

$$
\mathcal{Q}_{x x y} \approx \frac{2\left(\tau^{+}-\tau^{-}\right)}{3} u u^{\prime}
$$

Note that $\mathcal{Q}_{x x y} \approx 0$ when $\tau^{+}=\tau^{-}$. Upon substituting the above into (5.8) we find the tangential component of the deviatoric stress,

$$
T_{x x}=2 \tau^{+} \mu u u^{\prime \prime}-\frac{2 \rho \Lambda}{3}\left(u u^{\prime \prime}+\left(u^{\prime}\right)^{2}\right),
$$

where $\Lambda=\tau^{+} \tau^{-}$. At a no-slip boundary we still have $T_{x x}=-2 \rho \Lambda\left(u^{\prime}\right)^{2} / 3$.

The above analysis sheds further light on the results presented in Section 2. In terms of the moment basis, standard and non-equilibrium bounce-back impose $Q_{x x y}=0$ at the walls. This implies $\mathcal{Q}_{x x y}=2 u T_{x y}$. Non-equilibrium bounce-back also sets $u=0$, and thus $\mathcal{Q}_{x x y}=0$, which is consistent with equation (5.12) in the interior. For standard bounce-back, $u$ is generally small at the walls. This explains why the spurious oscillations are less severe for standard and non-equilibrium bounce back than for the original moment method and diffuse reflection when $R e=100$ and $M a=0.1 \sqrt{3}$. Note that the diffuse reflection condition places non-zero constraints on the third order moments. In particular, since $\Pi_{y y}=\Pi_{y y}^{(0)}$, the method imposes $Q_{x x y}=\rho / 9-R_{x x y y}$ at the boundary.

5.1. Analysis of a lattice Boltzmann equation with two relaxation times. The lattice Boltzmann algorithm with two relaxation times can be written concisely as

$$
\begin{aligned}
\bar{f}_{i}\left(\mathbf{x}+\boldsymbol{\xi}_{i}, t+1\right)=\bar{f}_{i}(\mathbf{x}, t) & -\frac{1}{\tau^{+}+1 / 2}\left[\frac{1}{2}\left(\bar{f}_{i}+\bar{f}_{\bar{i}}\right)-f_{i}^{(0+)}\right] \\
& -\frac{1}{\tau^{-}+1 / 2}\left[\frac{1}{2}\left(\bar{f}_{i}-\bar{f}_{\bar{i}}\right)-f_{i}^{(0-)}\right] .
\end{aligned}
$$

The discrete solutions of the TRT model for planar channel flow may be obtained in the same manner as the BGK case discussed in Section 3. Precisely the same solutions are obtained for 
$\mathbf{u}, T_{x y}$ and $T_{y y}$. The equation for the tangential stress is similar to equation (3.19) but with $\tau^{2}$ replaced by $\Lambda=\tau^{+} \tau^{-}$:

$$
\begin{aligned}
3(4 \Lambda-1)\left(T_{x x}^{j+1}-2 T_{x x}^{j}+T_{x x}^{j-1}\right)-12 T_{x x}^{j} & =4 \Lambda \rho\left(u_{j-1}^{2}-2 u_{j}^{2}+u_{j+1}^{2}\right) \\
& -16 \Lambda \tau^{+} \rho G\left(u_{j+1}+u_{j-1}-2 u_{j}\right) \\
& +6 \tau^{+} \rho G\left(u_{j+1}+u_{j-1}+2 u_{j}\right) .
\end{aligned}
$$

The numerical advantages of the TRT model are evident. We have already shown that there is no numerical slip error with the moment method, allowing the freedom to select the odd relaxation time based on stability requirements alone. Therefore we can set $\Lambda=1 / 4$ and adjust $\tau^{+}$according to the flow parameters while still satisfying the boundary conditions. We are also free to choose $\Lambda=1 / 6$, corresponding to the classic Padé compact finite difference scheme [30] without sacrificing the accuracy of our boundary conditions. The choice of "magic parameter" $\Lambda=1 / 6$ was found previously to eliminate fourth order spatial errors and thus said to be the optimal choice for computing diffusion [17]. Also, the error due to the body force discretisation can be elimianted for any $R e$ number by setting $\Lambda=3 / 16$. This is the value of the "magic parameter" that eliminates the numerical slip error of bounce-back [16]. However, although a fixed value of $\Lambda$ allows for an adjustable viscosity, it does not permit a variation in the coefficient $\mu \tau^{-}$. That is, a fixed $\Lambda$ will not allow for a variable relaxation of the largest contribution to the stress at $\mathcal{O}\left(\tau^{2}\right)$.

The method for imposing consistent Burnett stress boundary conditions first presented by Reis [36] and revisited in Section 4 is here modified for a TRT scheme. Since the no-slip condition is satisfied exactly for any collision operator with the moment-method we can still impose the tangential stress to be proportional to the square of the shear stress. But in light of equation (5.13), equation (4.4) is modified to

$$
T_{x x}=-\frac{2 \tau^{-}}{\mu} T_{x y}^{2} .
$$

The boundary condition for $\bar{\Pi}_{x x}$ now becomes

$$
\bar{\Pi}_{x x}=\frac{\rho}{3}+\frac{12 \tau^{-}}{\rho\left(2 \tau^{+}+1\right)} \bar{\Pi}_{x y}^{2},
$$

and the unknown $\bar{f}_{i}$ are found to be

$$
\begin{aligned}
& \bar{f}_{2}=\bar{f}_{1}+\bar{f}_{3}+\bar{f}_{4}+2\left(\bar{f}_{7}+\bar{f}_{8}\right)-\frac{\rho}{3}-\frac{12 \tau^{-}}{\rho\left(2 \tau^{+}+1\right)} \bar{\Pi}_{x y}^{2}, \\
& \bar{f}_{5}=-\bar{f}_{1}-\bar{f}_{8}+\frac{\rho}{6}+\frac{6 \tau^{-}}{\rho\left(2 \tau^{+}+1\right)} \bar{\Pi}_{x y}^{2}-\frac{\rho G}{4}, \\
& \bar{f}_{6}=-\bar{f}_{3}-\bar{f}_{7}+\frac{\rho}{6}+\frac{6 \tau^{-}}{\rho\left(2 \tau^{+}+1\right)} \bar{\Pi}_{x y}^{2}+\frac{\rho G}{4} .
\end{aligned}
$$

Figure 15 plots the tangential stress $T_{x x}$ at $R e=100$ and $R e=0.1$ using the TRT stress boundary conditions with $\Lambda=1 / 4$. The Mach number and grid resolution are $M a=0.1 \sqrt{3}$ and $n=33$, respectively. The PDE solution for the Burnett stress (5.13) and the discrete analytical solution (5.15) are also shown. The numerical prediction is completely free of spurious stress oscillations due to the consistent treatment of boundary values. Moreover, the agreement between the three solutions is excellent, which verifies our analysis and justifies 

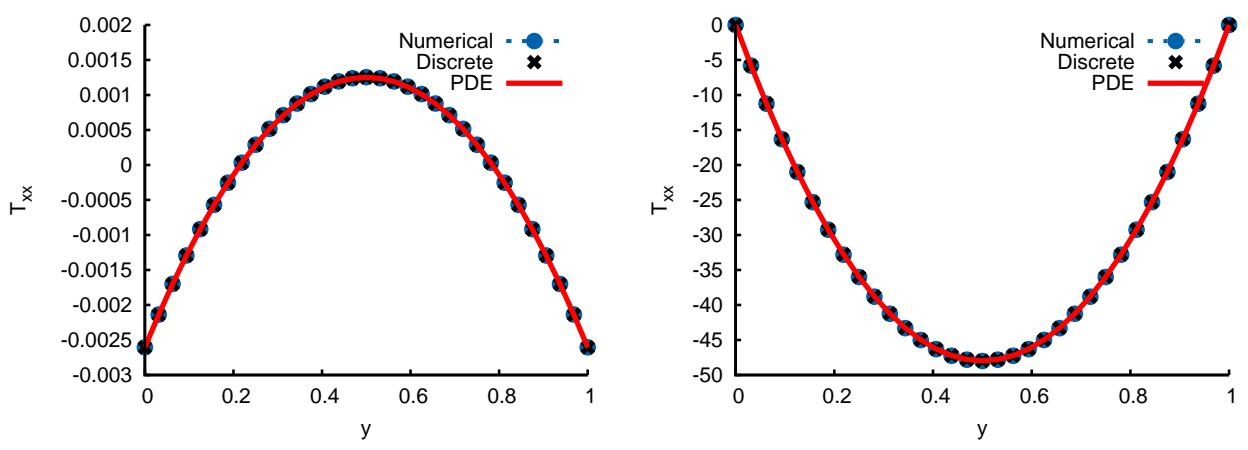

FIG. 15. (colour online) Plot of the PDE solution (5.13), discrete analytic solution (5.15) and numerical (computed TRT LBE) prediction of the tangential stress in an infinitely long planar channel using the TRT momentbased stress boundary conditions (5.18) with $\Lambda=1 / 4, M a=0.1 \sqrt{3}$ and $n=33$. Left: Re = 100; Right: $R e=0.1$.

the proposed boundary conditions. The computed solution form the LBE and and analytical solution of the LBE agree to machine precision and the difference between the LBE and the PDE solution differ by $\rho G^{2}(16 \Lambda-3)\left(1-\left(m^{j}+m^{n+1-j}\right) /\left(m+m^{n}\right)\right)$ at each grid point. The same trend has been observed when $\Lambda=1 / 6$ and all tested parameters. When $\Lambda=3 / 16$, the force error is removed.

Note that $K n=\mathcal{O}(1)$ for the right-hand plot of Figure 15, which is usually considered to be outside the realm of D2Q9 lattice Boltzmann models We must interpret this result with caution. The long relaxation time of the third order moment, as set by $\Lambda$, and the resulting negative parabolic profile of $T_{x x}$ may not be physically relevant. Be it a physical model or numerical artefact, the TRT constitutive equation for stress at second order exists and a failure to recognise it may result in a loss of computational accuracy, efficiency, and stability.

6. Discussion. Moment-based boundary conditions for the lattice Boltzmann equation usually assume that the tangential component of the deviatric stress vanishes at solid no-slip walls, as is the case in the Navier-Stokes equations. However, even though the D2Q9 model cannot capture kinetic effects in the velocity field, the deviatoric stress does include non-zero contributions at $\mathcal{O}\left(\tau^{2}\right)$ which coincide with the Burnett stress for isothermal planar channel flow. The neglect of these manifests in prominent oscillations in the computed solution of the stress, jeopardising the numerical stability and accuracy of the algorithm. This article has analysed the stress field as modelled by BGK and TRT lattice Boltzmann equations in planar channel with Navier-Stress and Burnett conditions to better understand the lattice Boltzmann deviatoric stress.

In Section 3 we followed He et al. [25] and analytically solved the BGK lattice Boltzmann equation in planar channel flow for both the velocity and stress fields. The momentbased method was shown to give the exact solution for the velocity using the minimum number of grid points $(n=3 \Longrightarrow \Delta x=1 / 2)$. The analytic solution for the tangential stress highlights the incompatibility of the Navier-Stokes moment-based boundary conditions which, by forcing $T_{x x}=0$, includes an inconsistent homogenous contribution and causes rapid oscillations in the computations when $\tau<1 / 2$. The Burnett boundary condition of Reis [36] for the deviatoric stress was revisited in Section 4 and modified for TRT schemes in Section 5. This method is fully local in space and time and inherits all the computational advantages of the lattice Boltzmann algorithm. Moreover, it eliminates the spurious 
oscillations in the stress field and the exact agreement between the solution of the reccurence relation and the numerical simulations confirmed our analysis. The small discrepancy between the LBE and discrete Boltzmann PDE solution at $\mathcal{O}\left(\tau^{2}\right)$ has been identified and shown to be due to the space-time discretisation of the force term.

The analytical solution reveals further numerical characteristics of the lattice Boltzmann equation. Equation (3.19) defines a tri-diagonal matrix for the deviatoric stress. For the specific value $\tau^{2}=1 / 6$ this difference equation corresponds to a fourth-order compact finite difference scheme (Padé scheme) for second order derivatives at gridpoints [30]. This is precisely the apparently "optimal" relaxation time found by Holdych et al. [26]. The choice $\tau^{2}=1 / 4$, on the other-hand, can be seen to enhance the numerical stability of the algorithm since it eliminates the recurrence in equation (3.19). This most stable value of $\tau$ for the BGK model is the basis of the lattice kinetic scheme of Inamuro [28]. The error due to the force vanishes when $\tau^{2}=3 / 16$. In this case, the LBE solution agrees with the PDE solution to machine precision.

The BGK model with equilibria defined by equation (1.4) does not permit the freedom to choose the relaxation time based on numerical considerations since $\tau$ is defined by the Reynolds number. In Section 5 we repeated our analysis with the two relaxation time model $[19,10]$. Here it was shown that the numerical characteristics are governed by the product of the odd and even relaxation times, $\Lambda=\tau^{+} \tau^{-}$, as discussed in the seminal work [10,17]. Now for any Reynolds (and Mach) number, one may eliminate the stress recurrence by choosing $\Lambda=1 / 4$ and adjusting the odd relaxation time accordingly (see also [10]). This may be useful for high Reynolds number flows on coarse domains. Similarly, setting $\Lambda=1 / 6$ yields a compact finite difference scheme for second order derivatives, which may be advantageous for diffusion-dominated flows. The error due to the force discretisation vanishes when $\Lambda=$ $3 / 16$, and this is likely to be a good choice for flows that are dominated, by the body force. Moreover, this is the value of the so-called "magic parameter" that eliminates the numerical slip error of bounce-back and yields a consistent algorithm [16]. However, one does not have the freedom to adjust the relaxation rate of the dominant contribution to the deviatoric stress with a favourable value of $\Lambda$. Thus the analysis has shed further light on the structure of the D2Q9 lattice Boltzmann algorithm, the influence of the relaxation times on the numerics, and the $\mathcal{O}\left(\tau^{2}\right)$ Burnett contributions to the stress at boundaries.

Acknowledgements. The author would like to acknowledge and thank Prof. Paul J. Dellar for his many helpful remarks and our discussions related to this work - they were always inspiring. This research forms part of the actives of the UK Consortium on Mesoscopic Engineering Science (UKCOMES) [grant number EP/L00030X/1].

\section{REFERENCES}

[1] R. Allen AND T. ReIS, Moment-based boundary conditions for lattice Boltzmann simulations of natural convection in cavities, Prog. Comput. Fluid. Dy., 16 (2016), pp. 216-231.

[2] S. Ansumali And I. V. Karlin, Kinetic boundary conditions in the lattice Boltzmann method, Phys. Rev. E., 66 (2002), p. 026311.

[3] S. BennetT, A lattice Boltzmann model for diffusion of binary gas mixtures, $\mathrm{PhD}$ thesis, University of Cambridge, Cambridge, UK, 2010. https://www.repository.cam.ac.uk/bitstream/handle/1810/226851/ ThesisHardcopy.pdf.

[4] S. BENNETT, P. ASINARI, AND P. J. DellaR, A lattice Boltzmann model for diffusion of binary gas mixtures that includes diffusion slip, Int. J. Numer. Meth. Fluids, 69 (2012), pp. 171-189.

[5] M. Bouzidi, M. Firdaouss, AND P. LALlemand, Momentum transfer of a Boltzmann-lattice fluid with boundaries, Phys. Fluids., 13 (2001), pp. $3452-3459$.

[6] J. E. BROADWELL, Study of rarefied shear flow by the discrete velocity method, J. Fluid. Mech., 19 (1963), pp. 401-414.

[7] C. Cercignani, Rarefied Gas Dynamics: From Basic Concepts to Actual Calculations, Cambridge Univer- 
sity Press, Cambridge, UK, 2000.

[8] S. Chapman AND T. G. Cowling, The Mathematical Theory of Non-Uniform Gases, Cambridge University Press, Cambridge, UK, 1970.

[9] P. J. DELlAR, Lattice Boltzmann formulation for linear viscoelastic fluids using an abstract second stress, SIAM J. Comput., 36 (2014), pp. A2507-A2532.

[10] D. D'HUMières AND I. GINZBURG, Viscosity independent numerical errors for lattice Boltzmann models: From recurrence equations to "magic" collision numbers, Comput. Math. Applic., 58 (2009), pp. 823840.

[11] D. D'Humières AND P. LALlemand, Numerical simulations of hydrodynamics with lattice gas automata in two dimensions, Complex Syst., 1 (1987), pp. 599-632.

[12] F. Dubois, Equivalent partial differential equations of a lattice Boltzmann scheme, Comp. Math. Appl., 55 (2008), pp. 1441-1449.

[13] O. FilipPova AND D. HÄNEL, Grid refinement for lattice-bgk models, J. Comp. Phys., 147 (1998), pp. 219228.

[14] L. García-Colín, R. Velasco, And F. URiBe, Beyond the Navier-Stokes equations: Burnett hydrodynamics, Phys. Rep., 465 (2008), pp. 149-189.

[15] R. Gatignol, Kinetic theory boundary conditions for discrete velocity gases, Phys. Fluids, 20 (1977), pp. 2022-2030.

[16] I. GINZBOURG AND M. P. ADLER, Boundary flow condition analysis for the three-dimensional lattice Boltzmann model, J. Phys. II, 4 (1994), pp. 191-214.

[17] I. GinZBurg, D. D'Humières, AND A. KuZmin, Optimal stability of advection-diffusion lattice Boltzmann models with two relaxation times for positive/negative equilibrium, J. Stat. Phys., 139 (2010), pp. 1090-1143.

[18] I. Ginzburg, F. Verhaeghe, And D. D'Humières, Study of simple hydrodynamic solutions with the two-relaxation-times lattice Boltzmann scheme, Commun. Comput. Phys., 3 (2008), pp. 519-581.

[19] I. Ginzburg, F. Verhaeghe, AND D. D'Humières, Two-relaxation-time lattice Boltzmann scheme: About parametrization, velocity, pressure and mixed boundary conditions, Commun. Comput. Phys., 3 (2008), pp. 427-478.

[20] H. GRAD, On the kinetic theory of rarefied gases, Commun. Pure Appl. Maths, 2 (1949), pp. 331-407.

[21] N. G. HADJICONSTANTINOU, The limits of navier-stokes theory and kinetic extensions for describing smallscale gaseous hydrodynamics, Phys. Fluids, 18 (2006), p. 111301.

[22] A. Hantsch, T. REIS, AND U. Gross, Moment method boundary conditions for multiphase lattice Boltzmann simulations with partially-wetted walls, J. Comput. Multiphase Flows, 7 (2015), pp. 1-14.

[23] X. He And L. S. Luo, Theory of the lattice Boltzmann method: From the Boltzmann equation to the lattice Boltzmann equation, Phys. Rev. E, 56 (1997), pp. 6811-6817.

[24] X. He, X. Shan, AND G. D. Doolen, Discrete Boltzmann equation model for nonideal gases, Phys. Rev. E, 57 (1998), p. R13.

[25] X. Y. He, Q. S. Zou, L. S. LuO, AND M. DEMBO, Analytic solutions of simple flows and analysis of nonslip boundary conditions for the lattice Boltzmann BGK model, J. Statist. Phys., 87 (1997), pp. 115-136.

[26] D. J. Holdych, D. R. Noble, J. H. Georgiadis, and R. O. Buckius, Truncation error analysis of lattice Boltzmann methods, J. Comput. Phys., 595-619 (2004), p. 193.

[27] E. ICKenberRy AND C. TRUesdell, On the pressures and the flux of energy in a gas according to Maxwell's kinetic theory, $i$, J. Rational Mech. Anal., 1 (1956), pp. 1-54.

[28] T. InAmURo, A lattice kinetic scheme for incompressible viscous flow with heat transfer, Phil. Trans. R. Soc. A, 360 (2002), pp. 477-484.

[29] T. LEE AND C. L. LIN, Rarefaction and compressibility effects of the lattice-Boltzmann-equation method in a gas microchannel, Phys. Rev. E., 71 (2005), p. 046706.

[30] S. K. LELE, Compact finite difference schemes with spectral-like resolution, J. Comput. Phys., 103 (1992), pp. 16-42.

[31] L. S. LuO, Unified theory of lattice Boltzmann models for nonideal gases, Phys. Rev. Lett., 81 (1998), pp. 1618-1621.

[32] J. C. MAXWELL, On stresses in rarified gases arising from inequalities of temperature, Phil. Trans. Roy. Soc. Lond., 170 (1879), pp. 231-256.

[33] S. Mohammed, D. GRAham, AND T. ReIs, Assessing moment-based boundary conditions for the lattice Boltzmann equation: A study for dipole-wall collisions, Comput. Fluids, 176 (2018), pp. 79-96.

[34] C. PAN, L. S. LUO, AND C. T. MILLER, An evaluation of lattice Boltzmann schemes for porous medium flow simulation, Comput. Fluids, 35 (2006), pp. 898-909.

[35] Y. H. Qian, D. D'Humières, AND P. LALlEmand, Lattice BGK models for the Navier-Stokes equation, Europhys. Lett., 17 (1992), p. 479.

[36] T. REIS, Burnett order stress and spatially-dependent boundary conditions for the lattice Boltzmann method, Communications in Computational Physics, 27 (2020), pp. 167-197.

[37] T. REIS AND P. J. DELlAR, Lattice Boltzmann simulations of pressure-driven flows in microchannels using 
Navier-Maxwell slip boundary conditions, Phys. Fluids, 24 (2012), p. 112001.

[38] M. SbRagaglia AND S. SUCCI, Analytical calculation of slip flow in lattice Boltzmann models with kinetic boundary conditions, Phys. Fluids, 17 (2005), p. 093602.

[39] X. Shan AND X. He, Discretization of the velocity space in the solution of the boltzmann equation, Phys. Rev. Lett, 80 (1998), pp. 65-68.

[40] Z. B. SinNAH, D. GRAHAM, AND T. ReIS, Lattice Boltzmann modelling of pulsatile flow using moment boundary conditions, in Proceedings of ECCM ECFD 2018, ECCOMAS, 2018.

[41] Y. Sone, Kinetic Theory and Fluid Dynamics, Birkhäuser, Boston, USA, 2002.

[42] H. STRUChtRUP, Macroscopic Transport Equations for Rarefied Gas Flows, Springer, Heidelberg, Germany, 2005.

[43] S. SUCCI, Mesoscopic modeling of slip motion at fluid-solid interfaces with heterogeneous catalysis, Phys. Rev. E, 89 (2002), p. 064502.

[44] G. H. TANG, W. Q. TAO, AND Y. L. HE, Lattice Boltzmann method for gaseous microflows using kinetic theory boundary conditions, Phys. Fluids., 17 (2005), p. 058101.

[45] C. TRUESDELL, On the pressures and the flux of energy in a gas according to Maxwell's kinetic theory, ii, J. Rational Mech. Anal., 1 (1956), pp. 55-127.

[46] F. VerhaEghe, L. S. LuO, AND B. Blanpain, Lattice Boltzmann modeling of microchannel flow in slip flow regime, J. Comput. Phys., 228 (2009), pp. 147-157.

[47] W. A. Yong AND L. S. Luo, Accuracy of the viscous stress in the lattice Boltzmann equation with simple boundary conditions, Phys. Rev. E, 86 (2012), p. 065701R.

[48] D. YU, R. MEI, AND W. ShYY, A unified boundary treatment in lattice boltzmann method, in 41st Aerospace Sciences Meeting and Exhibit, 2003, p. 953

[49] W. P. Yudistiawan, S. Ansumali, And I. Karlin, Hydrodynamics beyond Navier-Stokes: The slip flow model, Phys. Rev. E, 78 (2008), p. 016705.

[50] Y. H. ZhanG, X. J. Gu, R. W, BARBER, AND D. R. Emerson, Capturing knudsen layer phenomena using a lattice Boltzmann model, Phys. Rev. E, 74 (2006), p. 046704.

[51] F. ZhaO, Optimal relaxation collisions for lattice Boltzmann methods, Comput. Math. Appl., 65 (2013), pp. $172-185$.

[52] W. ZHAO AND W. YONG, A family of single-node second-order boundary schemes for the lattice boltzmann method, arXiv preprint arXiv:1712.08288, (2017).

[53] Q. Zou AND X. HE, On pressure and velocity boundary conditions for the lattice Boltzmann BGK model, Phys. Fluids, 9 (1997), p. 1691. 